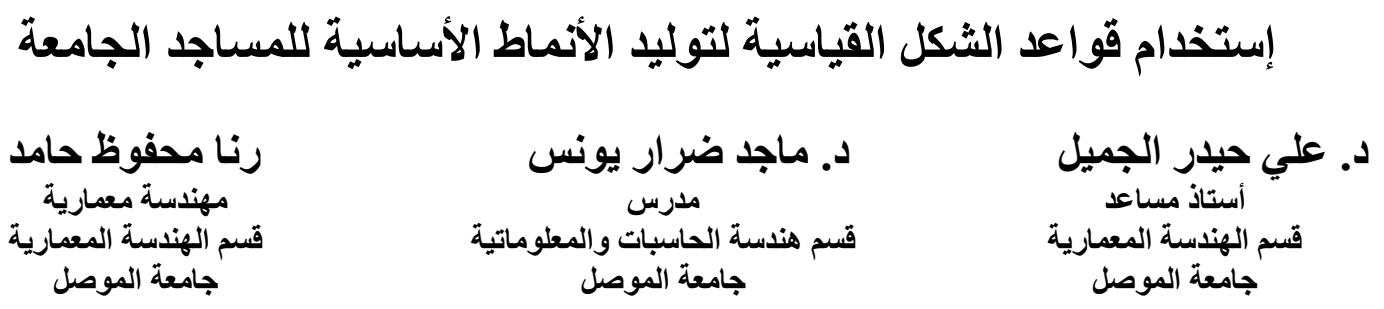

تعدُّ قواعد الثكل من المفاهيم الحديثة التي اكتسبث أهمية كبيرة في مجالات مختلفة لفوائدها المتعددة. وقد

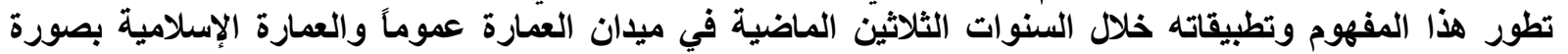

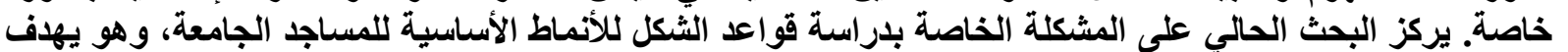

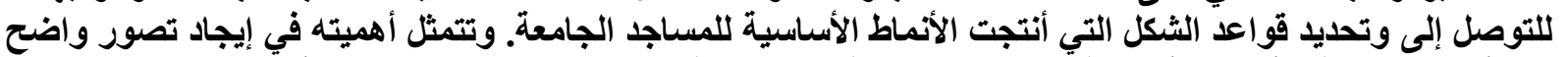

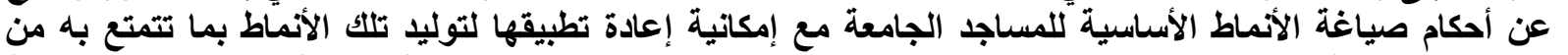

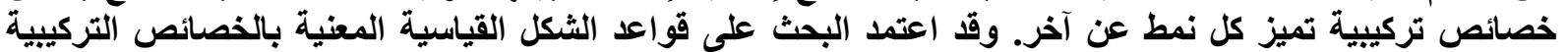

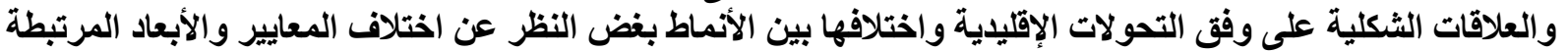

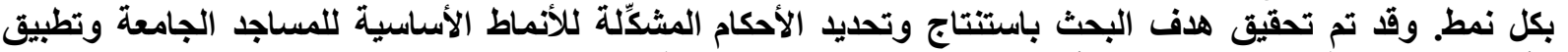
الأحكام المستتتجة في اشتثقاق هذه هذه الأنماط لاختبار إمكانياتها التطبيقية.

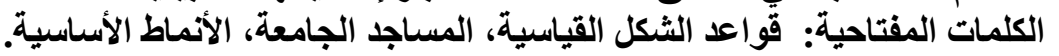

\title{
Using Standard Shape Grammars to Generate The Basic Types of Congregational Mosques
}

\author{
Dr. Ali H. Al-Jameel \\ Assistant Professor \\ Architectural EngineeringDpt. \\ Mosul University
}

\author{
Dr. Maged D. Younis \\ Senior Lecturer \\ Computer Engineering Dpt. \\ Mosul University
}

\author{
Rana M. Hamed \\ Architect \\ Architectural Engineering Dpt. \\ Mosul University
}

\begin{abstract}
Shape Grammars is among the recent concepts that have gained considerable significance for its multiple benefits for different fields. This concept with its applications had been evolved during the last thirty years within the field of architecture generally and Islamic architecture in particular. The main goal of the present research is to study the shape grammars of the congregational mosques basic types, analyzing them to conclude and define the shape rules that generate those types. Its significance comes from providing a more clear conception for the rules that had produced the basic types of congregational mosques with the possibility of reapplying the concluded rules to derive those types with their formal and syntactical properties. The research adopted (Standard Shape Grammar) which is interested in syntactical characteristics, formal relationships according to Euclidean transformations between different types regardless the different criteria and dimensions of each one. The research goal has been achieved by concluding the shape rules formulating the basic types of congregational mosques and reapplying the concluded rules to derive those types to test their potentialities for the generation process.
\end{abstract}

Keywords: Standard Shape Grammar, Congregational Mosques, Basic Types

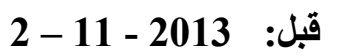

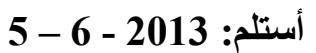


بنائية مختلفة للعمارة الإسلامية، فإنه

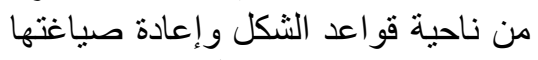

ه إكتسب مفهوم قو اعد الثكل في مجال العمـارة، منذ آن طرح لأول مرة في سبعينيات القرن العشرين، أهميـة كبيرة

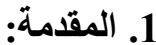

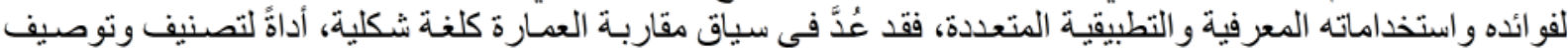

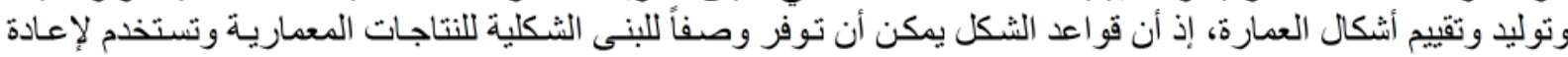

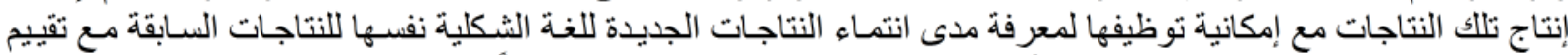

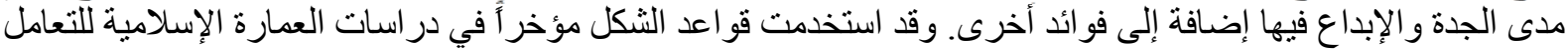

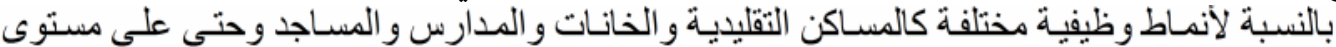
صر المعمارية كالزخرفة الإسلامية، ويأتي البحث الحالي إسهامة جديدة ضمن هذا السياق.

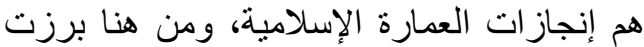

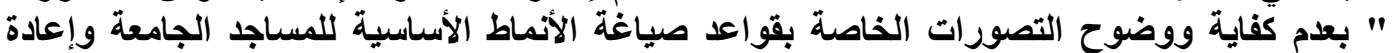

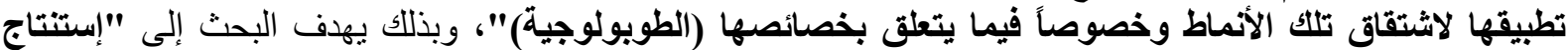

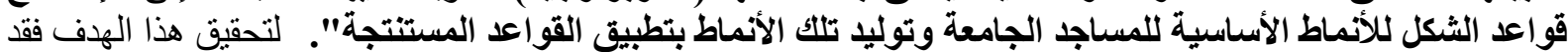

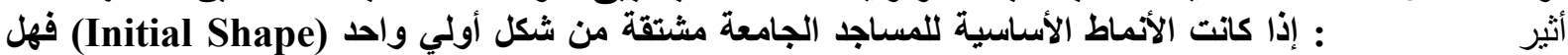

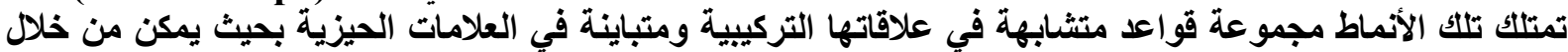

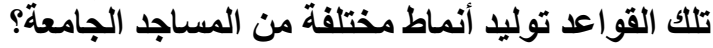

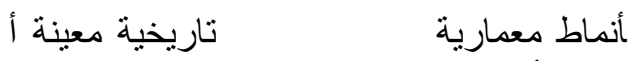

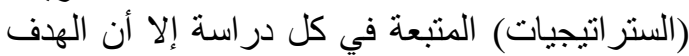

3. 3. إستعراض الاراسات السابقة: 1.3. دراسات قواعد الثكل في العمارة: الدر اسات المعماريد

تحليل النماذج المنتخبة و إيجاد قو اعدها ومن ثم نوليد النموذج الأصلي و عدد من النماذج الجديدة المنتمية لنفس النوع. وقد (Palladio)

(1978 Stiny \& Mitchell)

القواعد التحليلية وهي القواعد التي تستخدم لوصف وتحليل الطراز التاريخي للتصاميم المعمارية من خلال تحليل Queen (1987 Flemming)

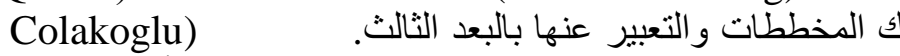

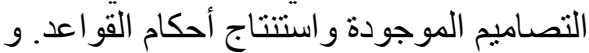

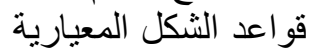

(Ann قو اعد الثكل لتوليد مساكن حياة (Hayat Houses) مستخدمة القواعد اعد التولية التحليلية والأصلية لتحليل $(2000$

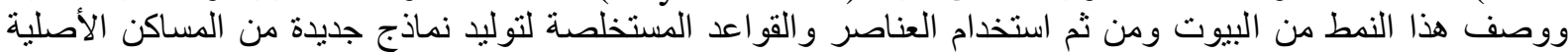

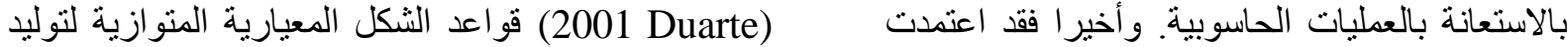

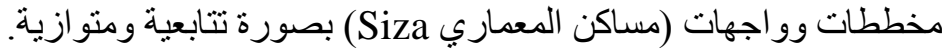

بتنوع الأنماط الوظيفية و الفتر ات الزمنية، وتئة

2.3. دراسات قواعد الثكل في العمارة الإسلامية: (1996 Cagdas) كمنهجية لتحليل وتوليد التصاميم (Gips) (2004 Ahmed \& Chase)

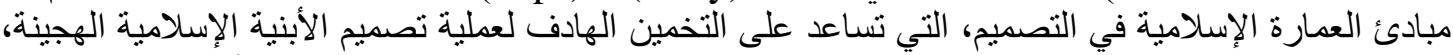

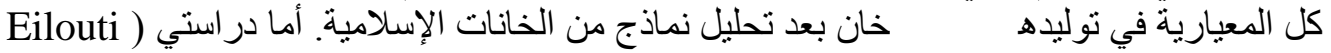

2007 \& Al-Jokhadar

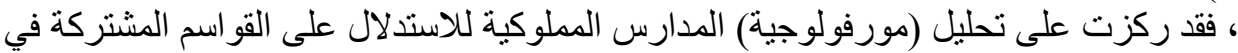

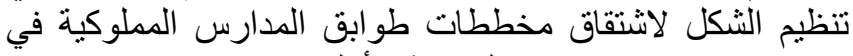
الجوانب الثكلية و التركيبية فيما بينها واستنباط إنياط

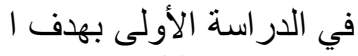
الدراسة الأولى، وتم في الدراسة الثانية إعادة صياغة فيانة

$$
\text { (2008 Sener \& Gorgul) }
$$

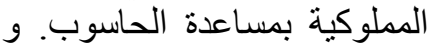

وتمثيل المبادئ التناسبية لكتل المساجد

لإعداد خوارزمية قواعد الثكل ول (Delphi-Based)

العثمانية (ستر اتيجية) العثمانية الكلاسيكية في الأبع 
التحول لاحقا. في حين تناولت در اسة (2009 Ulu \& Sener)

الأخرى تباعا، ويأتي بعدها اعتماد

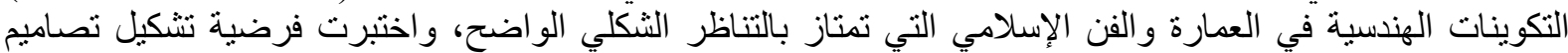

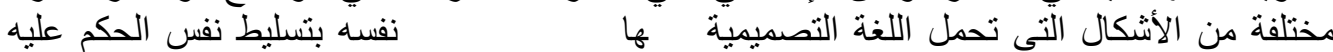
التناظر حول المحاور على مستوى الجزء أو الكل يعد من أهم السمات الأساسية التي تتمتع بها زخارف الته الفن الإسلامي

2011 AL- Kazzaz)

باستخدام قواعد الثكل كأداة تقييمية لقياس درجة إبداع التصاميم الهجينة المنولدة

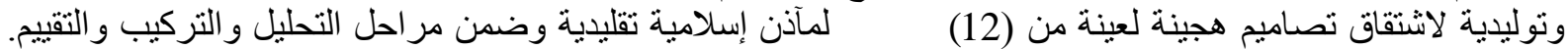

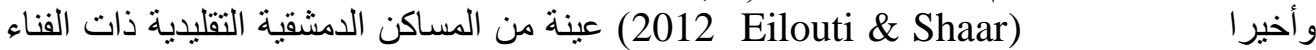

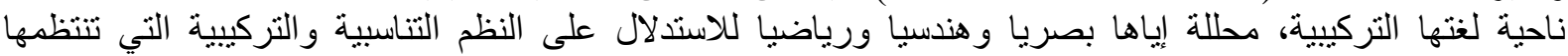
الثكل، التي بتطبيقها، مكن توليد و اشتقاق تصاميم نموذجية لنفس النمط.

\section{1.4. مفهوم قواعد الثكل في العمارة:

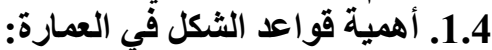

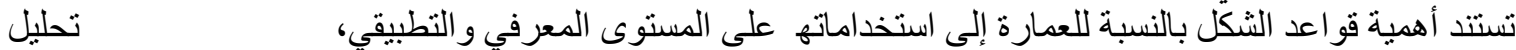

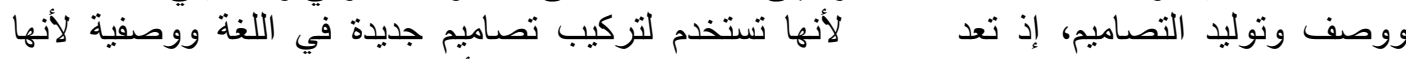
الموضوعية لثرح البنية الثكلية للتصاميم التي يتم توليدها، كانت التصاميم الجديدة تنتمي لنفس اللغة التصميمية (19) 2012 Duarte)

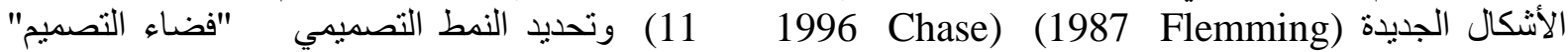

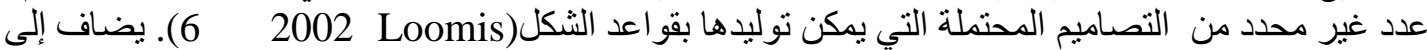

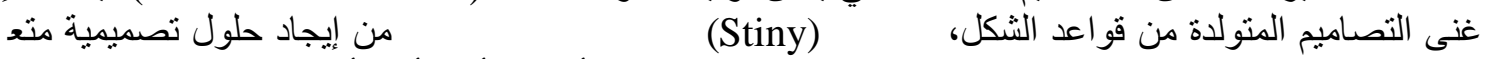

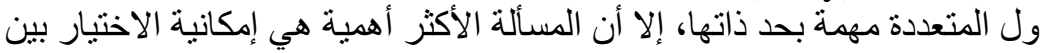

مختلف الحلول لغرض التوصل إلى أفضلها(1980b Stiny). س لن الن يحتاج

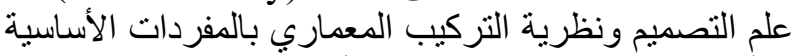

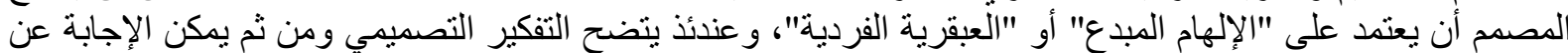

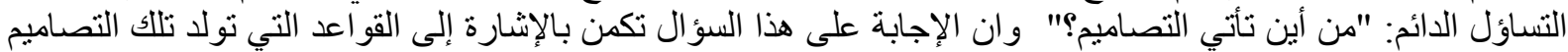
(1980b Stiny)

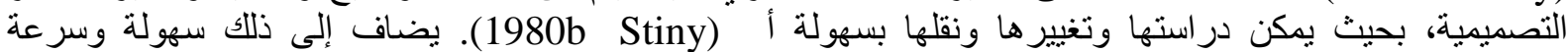

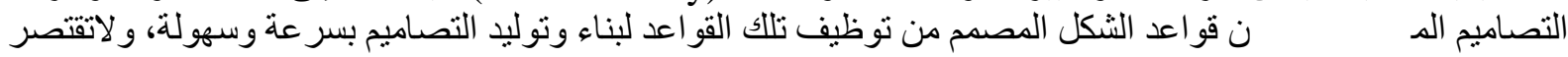

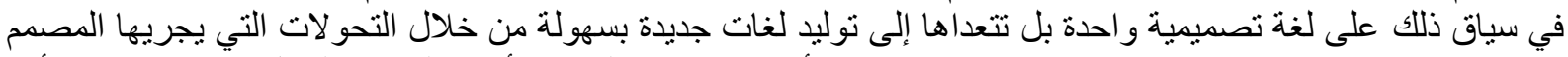
(ئم) 1981 Knight) .

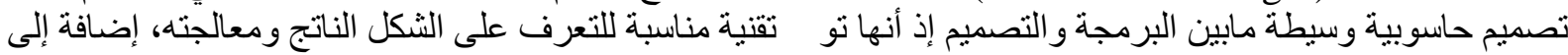

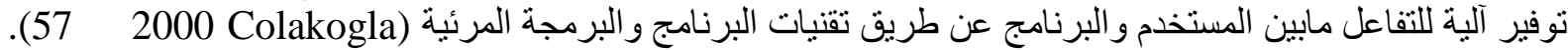

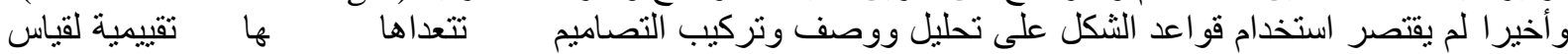
تصاميم المتولدة واعت المقارنة بينها (2011) Al-Kazzaz)

هي: (1980b Stiny)

2.4 2.4 عناصر مفهوم قواعد الشكل في العمارة:

L :Symbols .2

يتم من خلال الرموز أو ماتسمى العلامات (Labels) تمييز الحالات المختلفة للثكل، فالثنكل اله (Labeled Shape) (345 1980b Stiny).

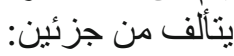
R

:Shape Rules .3

addition rules: (1) $A \rightarrow A+B$, مة يفصل بينها

(2) $B \rightarrow A+B$

subtraction rules: (3) $A+B \rightarrow A$, انية

سهمة بصبخة:

(4) $A+B \rightarrow B$. ترنيب الأشكال) فيما بينها، وتمثل العلاقات المكانية الأفكار

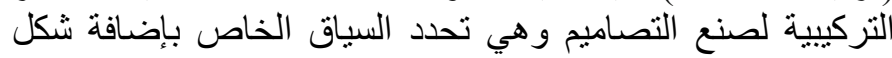


ح شكل من آخر بهدف إنشاء التصميم (1994 Knight 196 (706).

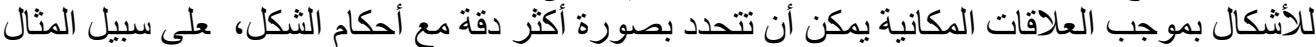

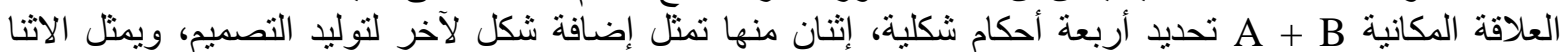

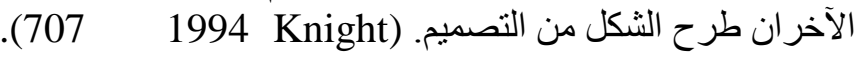
.I" : Initial Shape .4

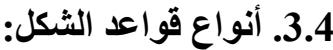

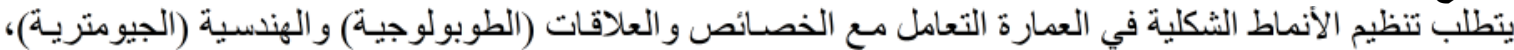

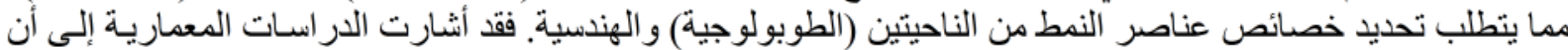

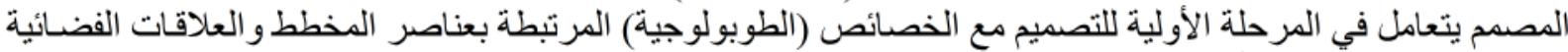

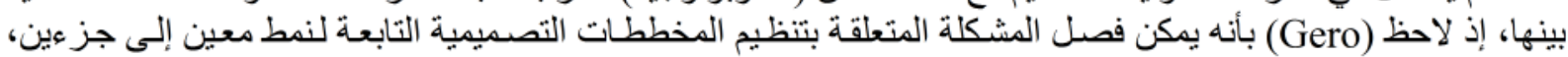

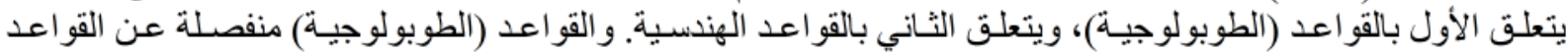

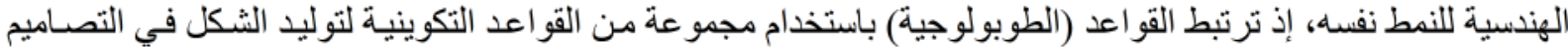

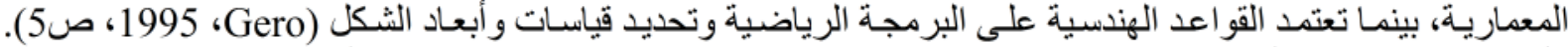

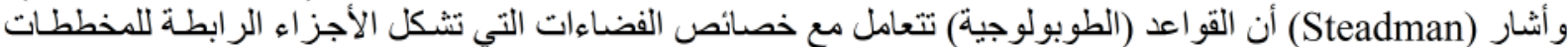

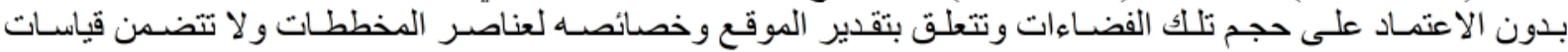

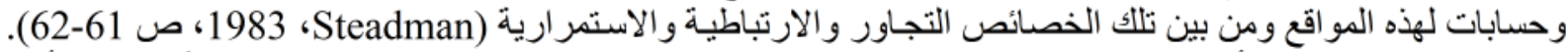

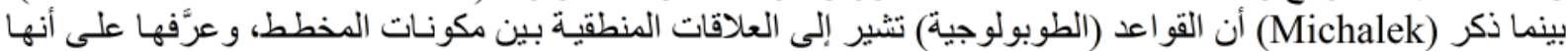

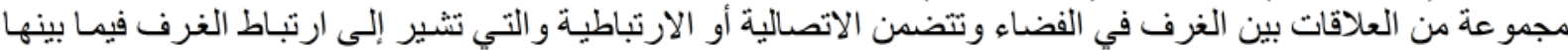

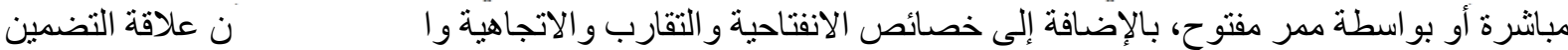

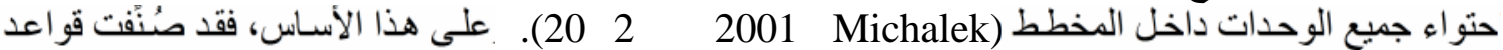
طبيعة الخصائص التصميمية إلى نو عين من القو اعد:

أولاً/ قو اعد الثكل القياسية ( Standard Shape Grammars):

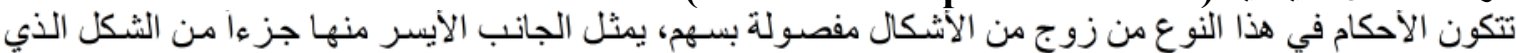

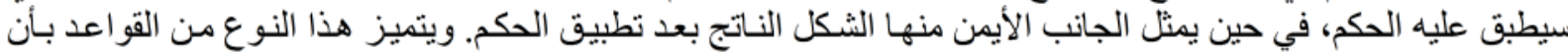

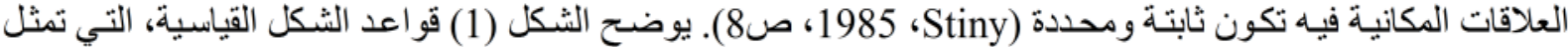

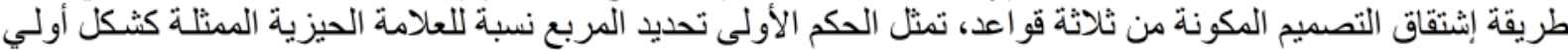
(Initial Shape)

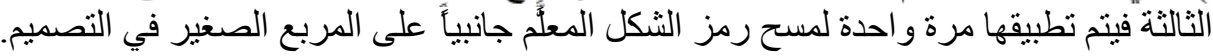

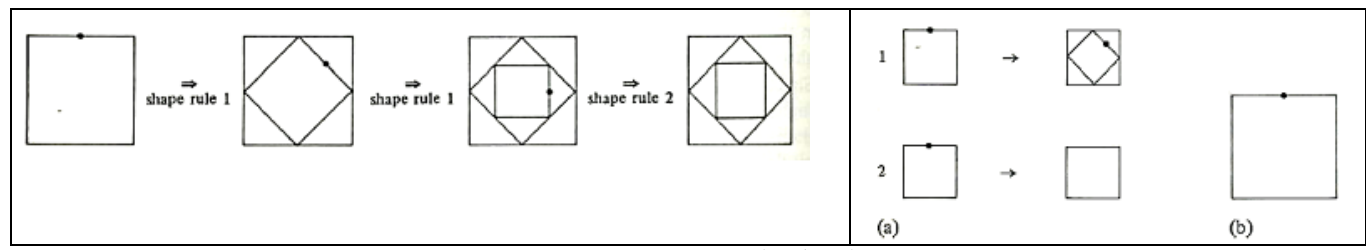

1980a Stiny) ق 19 اعد الثكل القياسية

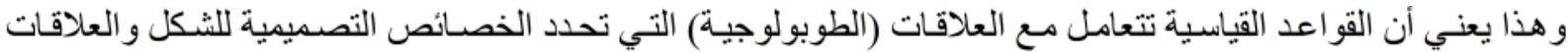

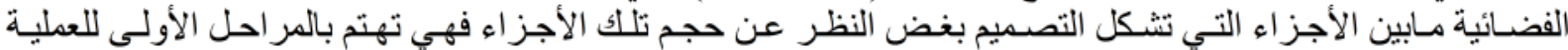

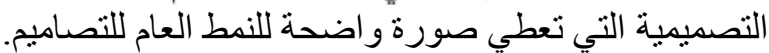

ثانياً/ قواعد الثكل المعيارية (Parametric Shape Grammars)

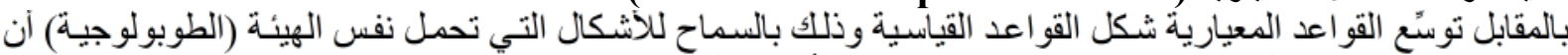

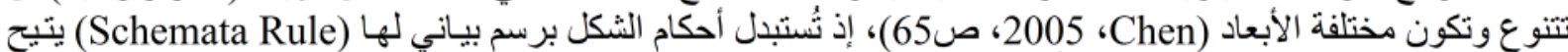

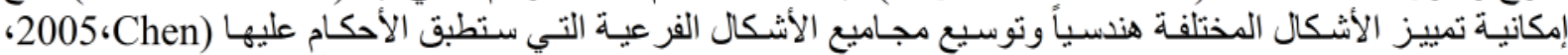

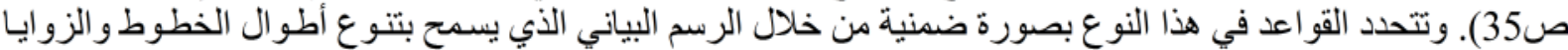

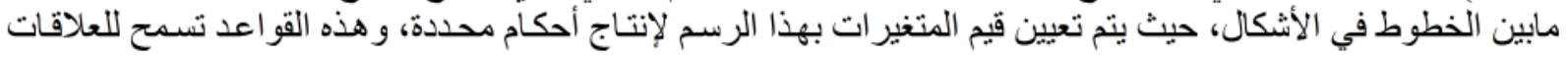




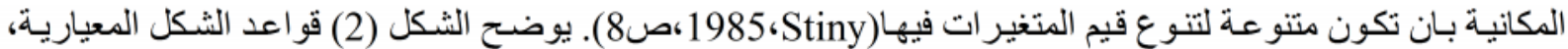

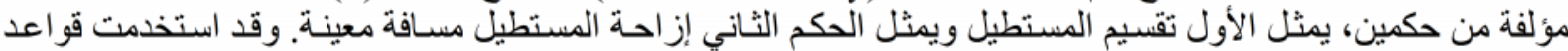

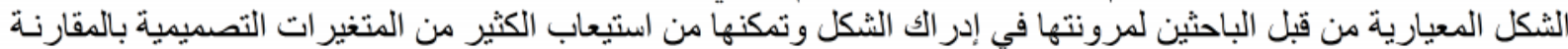

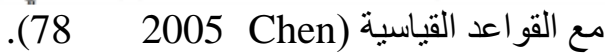

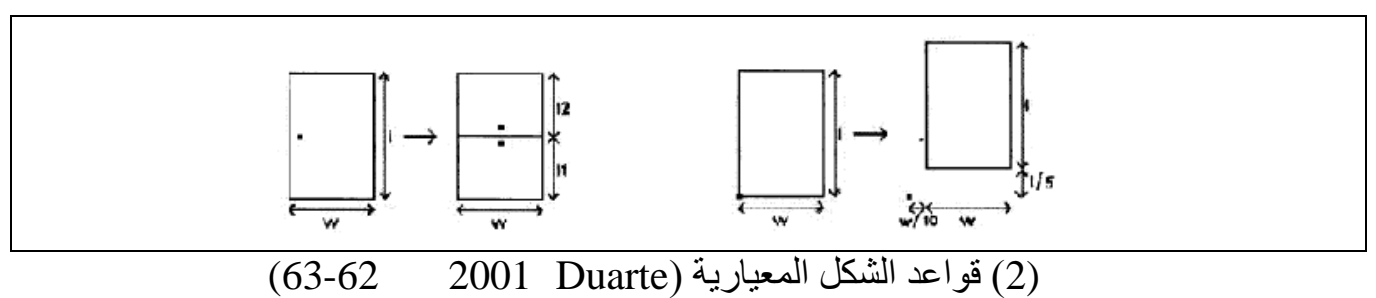

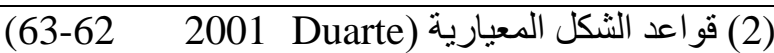

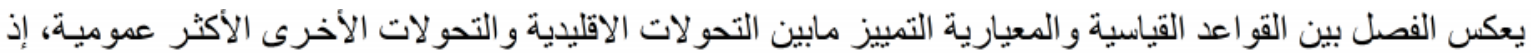

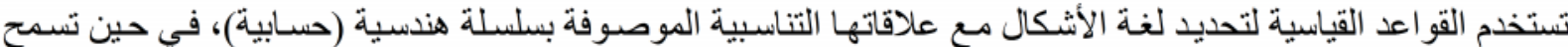

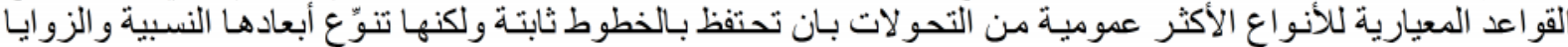

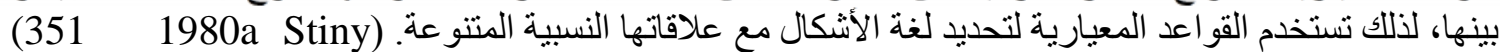

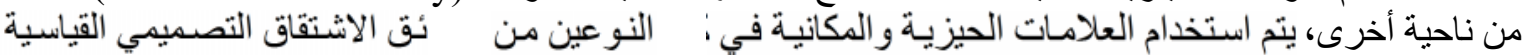

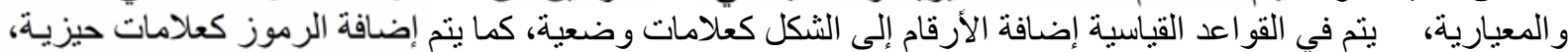

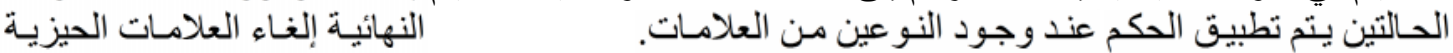
و الوضعية، كما يتم في القو اعد المعيارية استخدام العلامات الحيزية و المكانية ولكن بـ ة أوسع إذ تسمح للحكم بأن بطبَّق ضمن الحدود المعيارية للأشكال(218-217) 2006 Orsborn \& Others).

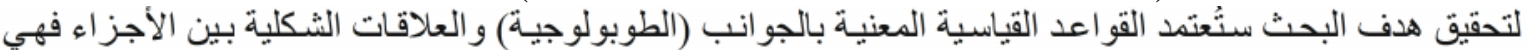

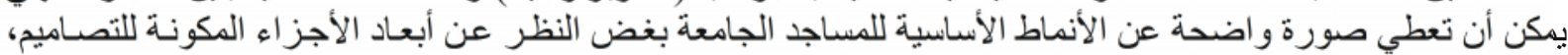

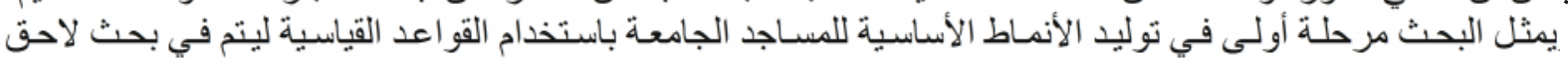
يارية المعنية إضافة للعلاقات الفضائية الجو انب الهندسية وأبعاد الأجزاء التصديمية.

5. منهجية وإجراءات البحث:

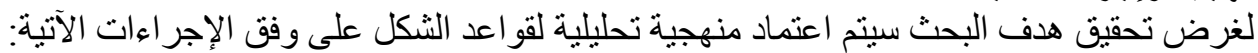

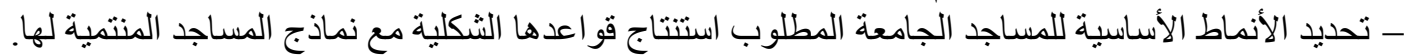

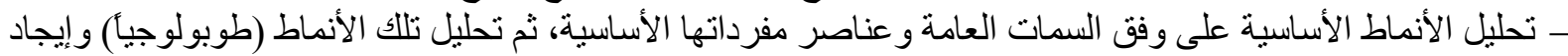
العلاقات بين عناصر مفرداتها الأساسية.

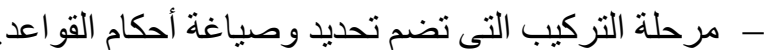

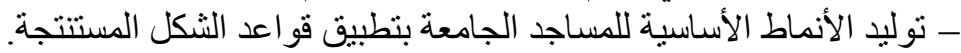

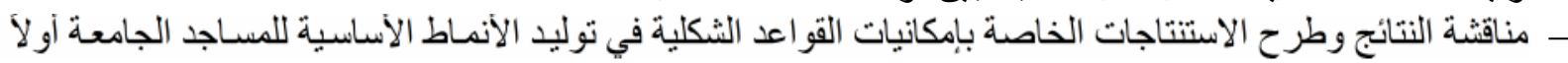
وماتعنيه بالنسبة لهدف

1.5 تصديد الأنماط الأساسية للمساجد الجامعة:

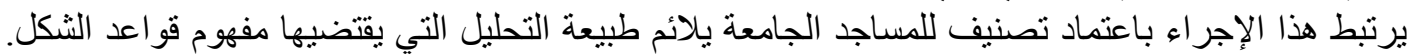

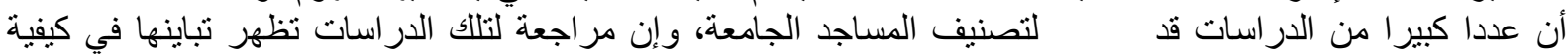

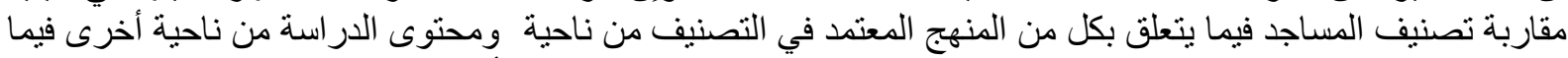

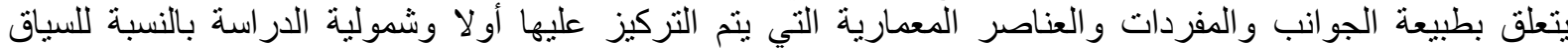

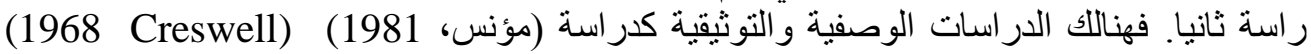

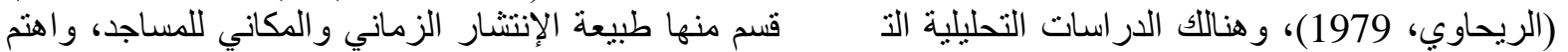
وتحليل هذه الخصائص المن قسم آخر بدر اسة خاصية أو مجموعة خصائص شكلية كانت أو تركيبية
) (1978 Michell

.(1994 Hillenbrand) (1988

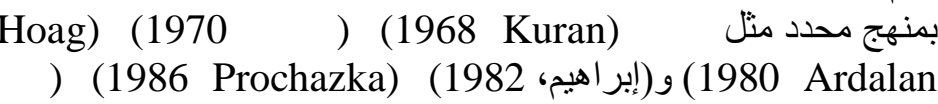

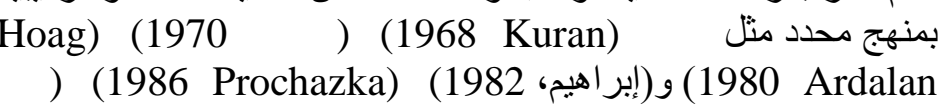


هذا البحث سيعتمد التصنيف الذي طرحته دراسة (الجميل و الجبوري و 2013)

(Unsupervised Pattern Recognition)

باستخدام طريقة تمبيز النمط لئة

هي:

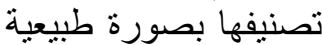
مقبب السقف غير ذي الفناء و طئه

(1) علما أن التحليل قد شمل عينة منتخبة من المساجد الجامعة المنتشرة على معظم السياق الزمه

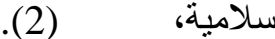

الأنماط الثكلية الأساسية للمساجد الجامعة المستتنجة من التحليل بطريقة تمييز النمط

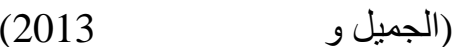

\begin{tabular}{|l|l|l|l|l|}
\hline & & النمط مقبب السقف غير & & \\
\hline \\
\hline 0
\end{tabular}

(2)

المساجد الجامعة المنتخبة للتحليل بطريقة تمبيز النمط

\begin{tabular}{|c|c|c|c|c|c|}
\hline \multicolumn{6}{|c|}{$(2013$} \\
\hline المساجد المنتخبة & الرمز & المساجد المنتخبة & 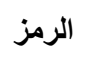 & المساجد المنتخبة & الرمز الرمز \\
\hline & $\mathrm{m} 25$ & & $\mathrm{~m} 13$ & & $\mathrm{~m} 1$ \\
\hline جامع سليم & $\mathrm{m} 26$ & مسجد أصفهان & $\mathrm{m} 14$ & & $\mathrm{~m} 2$ \\
\hline جامع يلدرم & $\mathrm{m} 27$ & & $\mathrm{~m} 15$ & & $\mathrm{~m} 3$ \\
\hline جامع السليمانية & $\mathrm{m} 28$ & مسجد كاليان & $\mathrm{m} 16$ & & $\mathrm{~m} 4$ \\
\hline & $\mathrm{m} 29$ & مسجد كولبايكان & $\mathrm{m} 17$ & & $\mathrm{~m} 5$ \\
\hline جامع شيهز اد & $\mathrm{m} 30$ & مسجد جوهر شاد & $\mathrm{m} 18$ & & m6 \\
\hline جامع السليمية & $\mathrm{m} 31$ & جامع بورصة الكبير & $\mathrm{m} 19$ & مسجد شامبانير & $\mathrm{m} 7$ \\
\hline & $\mathrm{m} 32$ & السلطان بايزيد الثاني & $\mathrm{m} 20$ & مسجد دلهي & $\mathrm{m} 8$ \\
\hline جامع الثريفلي & $\mathrm{m} 33$ & & $\mathrm{~m} 21$ & & $\mathrm{~m} 9$ \\
\hline جامع الملكة صفية & $\mathrm{m} 34$ & جامع أتيق علي بانشا & $\mathrm{m} 22$ & مسجد سفاريه & $\mathrm{m} 10$ \\
\hline & $\mathrm{m} 35$ & جامع ادرنه القديم & $\mathrm{m} 23$ & مسجد بيبي خانم & $\mathrm{m} 11$ \\
\hline جامع بايزيد الثاني & $\mathrm{m} 36$ & محمد باشـا اليوناني & $\mathrm{m} 24$ & & $\mathrm{~m} 12$ \\
\hline
\end{tabular}

2.5 2.5 مرحلة التحليل: 2.5

1.2.5 عناصر المفردات الأساسية للمساجد المنتخبة:

اختلاف الدر اسات المعمارية حول ماهية العناصر و المفردات المكونة

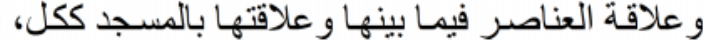

تباين تصنيفات

وأهمية تبن

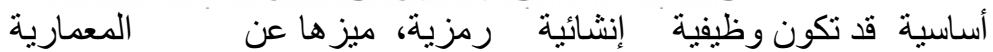

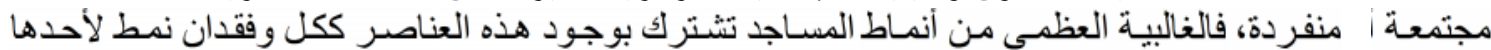

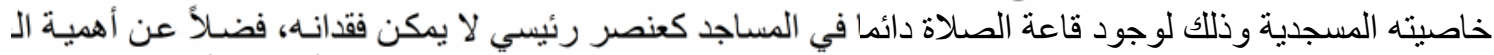

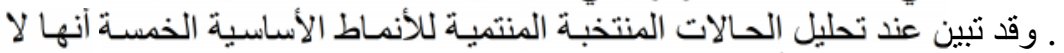

$$
\text { تخلو من العناصر الرئيد الآتية سواء نوفرت جميعها منهاوهي: }
$$




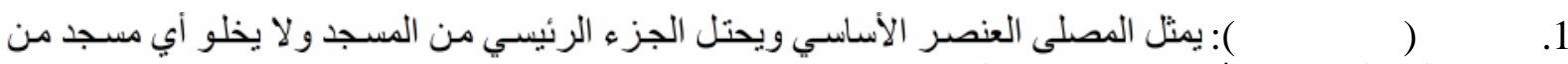

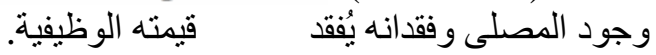

2. الفناء (صحن المسجد): يمثل الجزء غير المسقوف من المسجد، ويتخذ شكلا

فضاء ربط بين الفناء وحدود المسجد أ قد تفصل بين الفناء

الثانوية) لتمييز هاء عن الفضاء الرئيسي ألفئ
الثانوية ( ) ): تحيط

حث تسميتها (تحبط

2.2.5. التحليل الهندسي (الجيومتري) للعناصر الرئيسية المميزة لأنماط المساجد الجامعة:

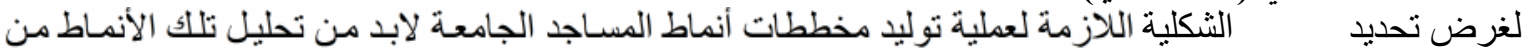

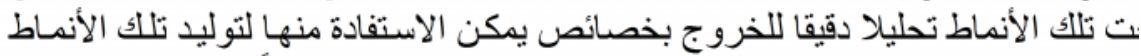

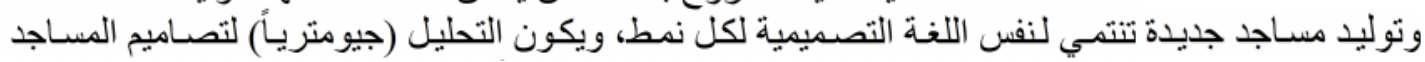
على هذا التحليل.

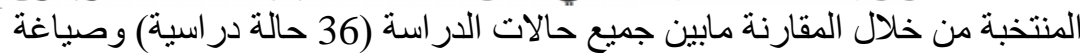

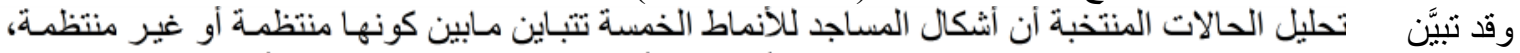

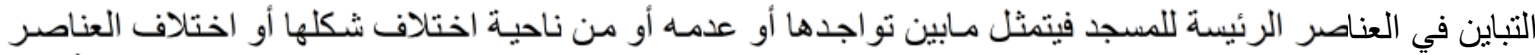

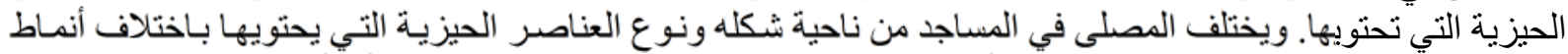

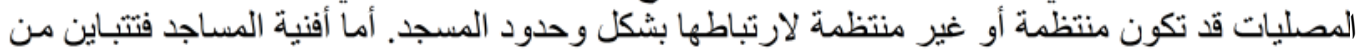

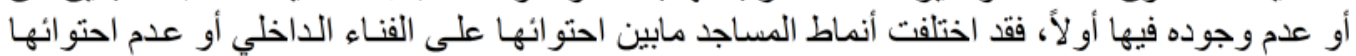
ناحية

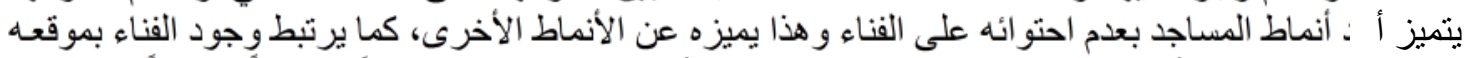

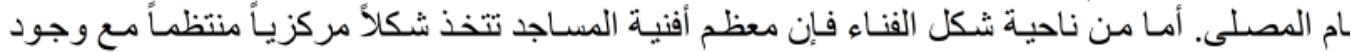

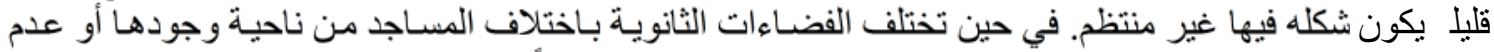

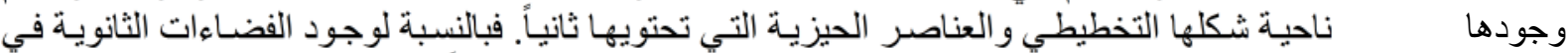

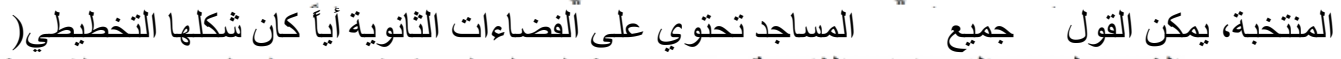

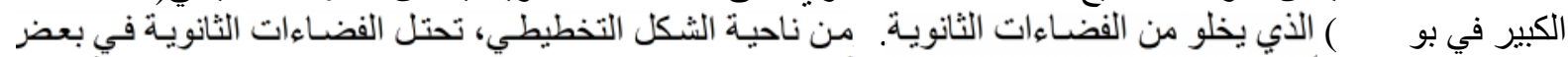

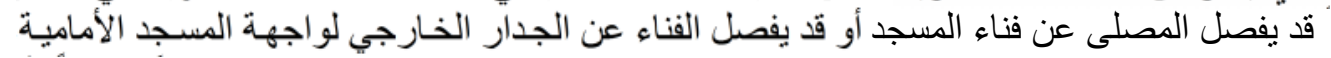

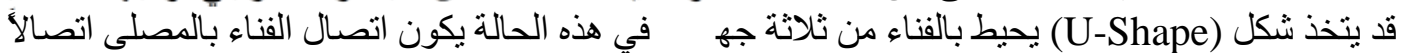

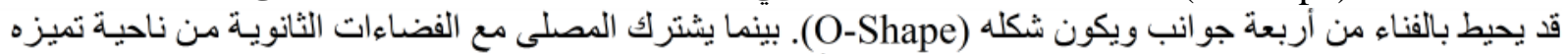

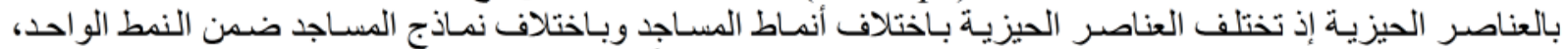

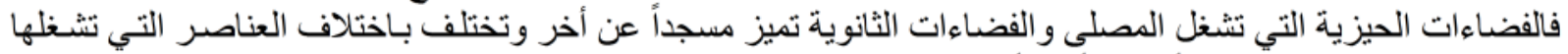

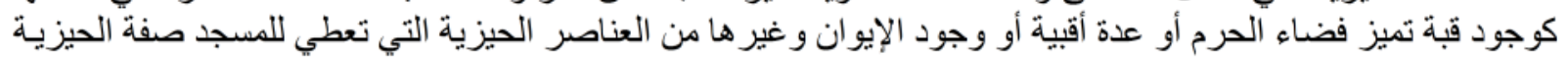

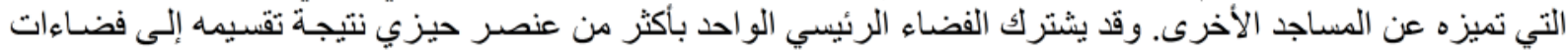

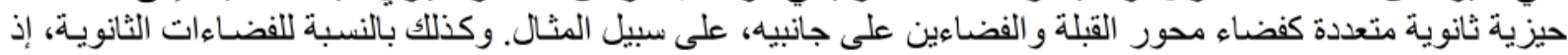

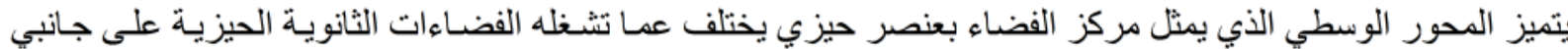

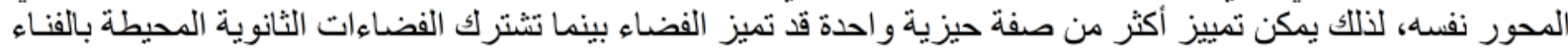

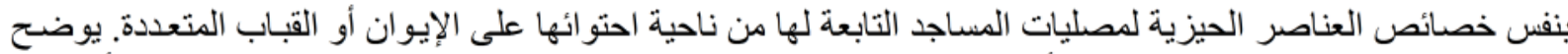

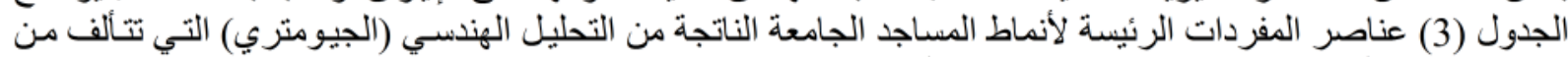

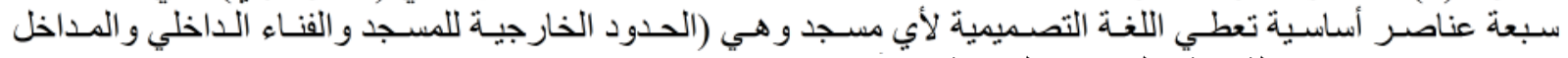
الثانوية العناصر الحيزية

(3)

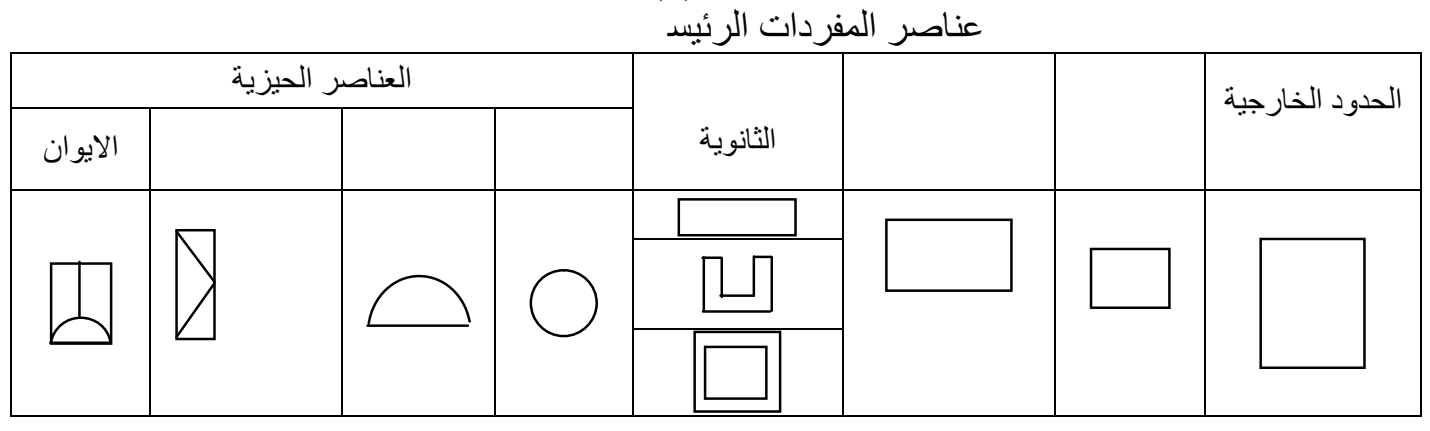


3.2.5 تحديد العلاقات الفضائية بين العناصر الرئيسة المميزة للأنماط الأساسية:

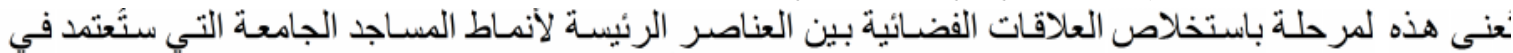

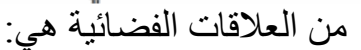

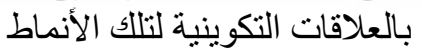

صباغة الأحكام الثكلية

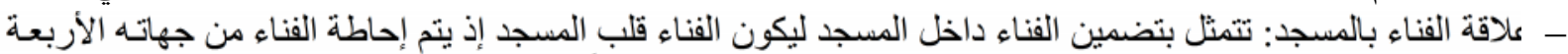

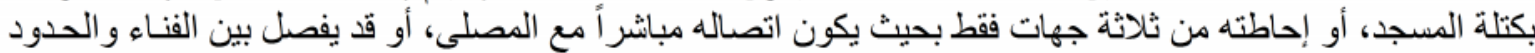

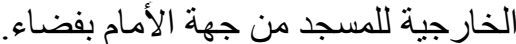
ـ علاقة المصلى بالمسجد: تتحدد هذه العلاقة من خلال تضمين المصلى داخل المسجد، حيث يحتل المصلى الجزء الخلفي

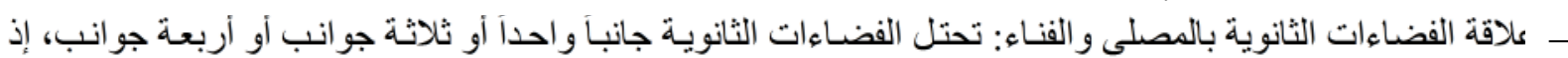

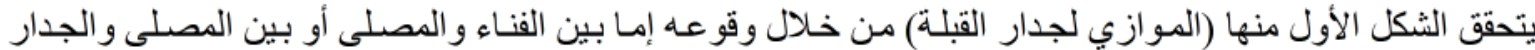

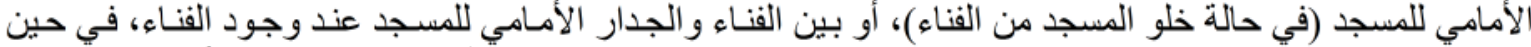
يتحقق الثكل الثاني (U-Shape) و الثكل الثالث (O-Shape) من خلال إضافة الأجزاء الثاء الثانوية متمثناً بالعلاقة مـابين

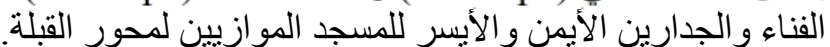

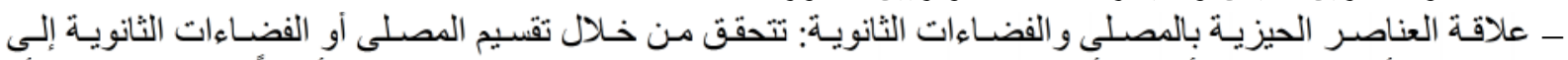

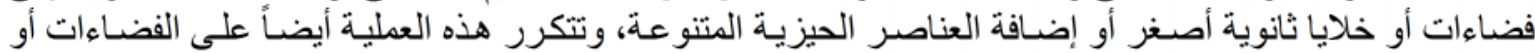

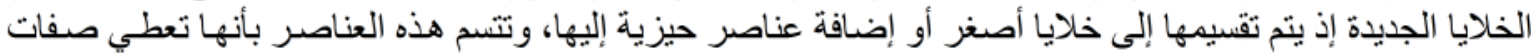

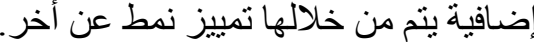

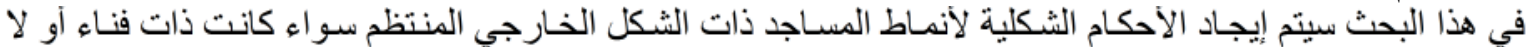

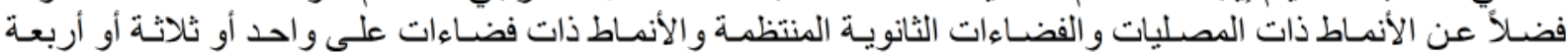

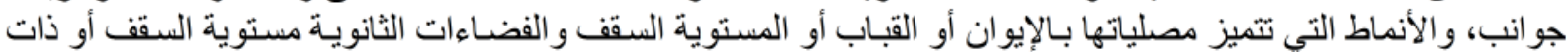

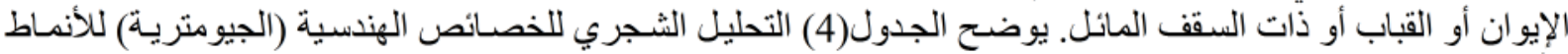
النماذج المنتخبة للتحليل و الدر اسة منها.

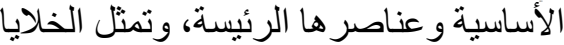

(4)التحليل الثجري للخصائص الهندسية (الجيومترية) لـ الاساسية و عناصر ها الرئيسة

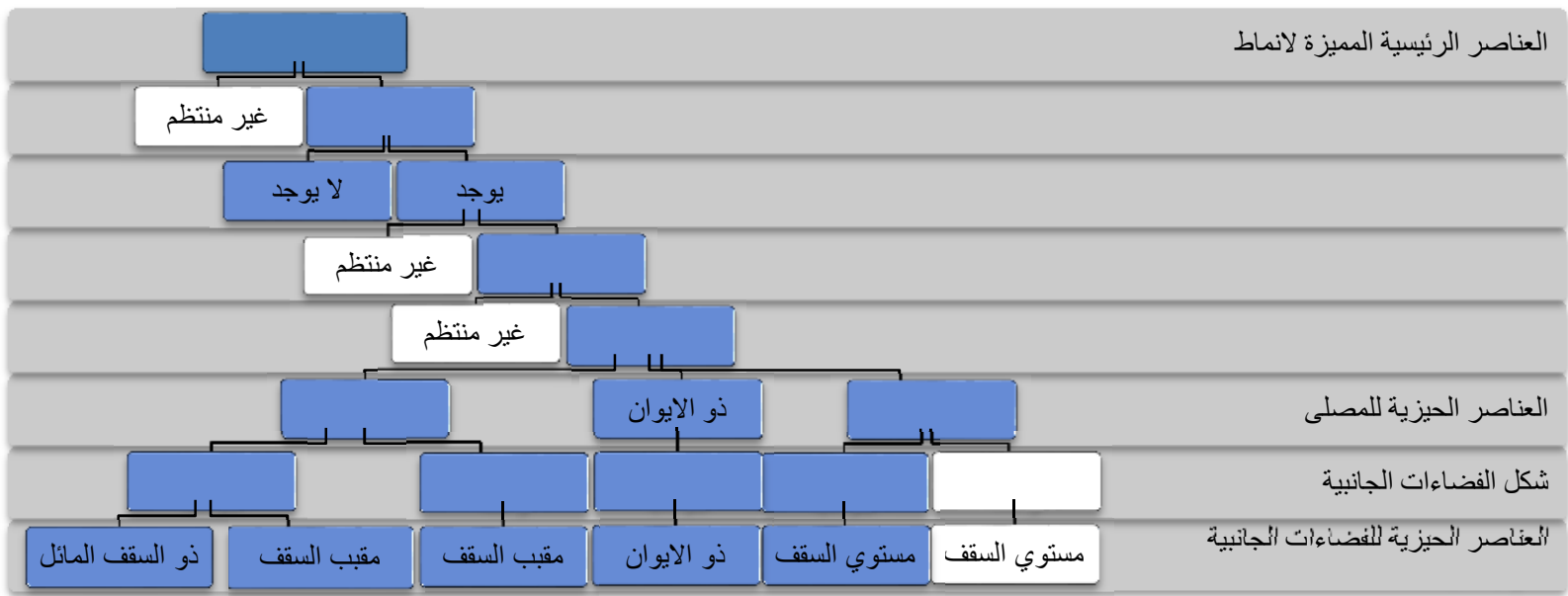

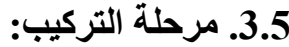

1.3.5 (ستراتيجيات) صياغة الأحكام الثكلية:

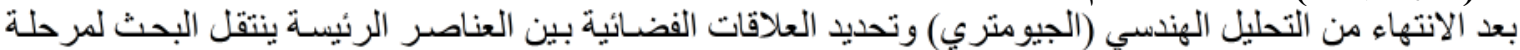

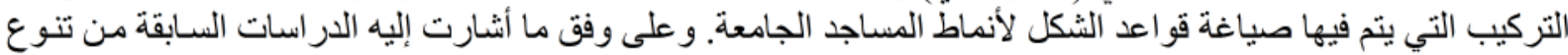

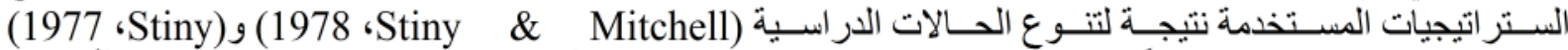

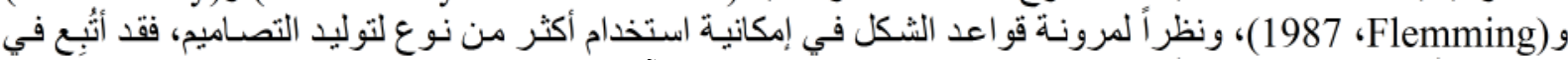

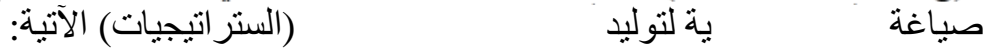

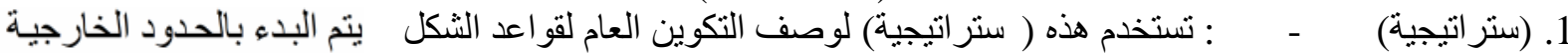
عناصر هـ حيزية، لذلك يتم الانتقال من الخارج 
2. (ستر اتيجية) : بعد تحديد الحدود الخارجية يتم إضـافة العنصر الأساسـي في تكوين العمارة الإسـلامية عامـة

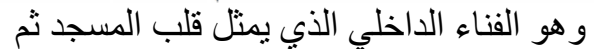

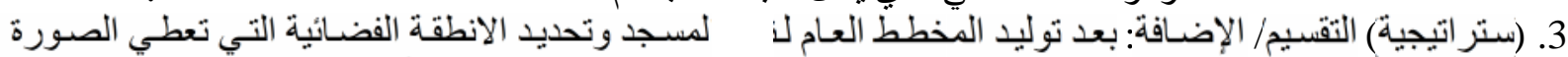

الأولية ينتقل البحث

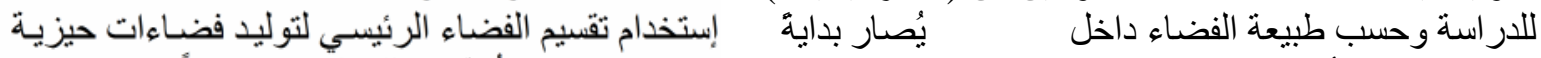
خلايا ثانوية أ الخلايا الثانوية المتولدة لحين صياغة التكوين الثامل. هذه ( ستر اتيجية)

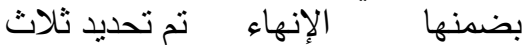

التي من خلالها بمكن تمييز نمط عن الإنهاء

لتوليد

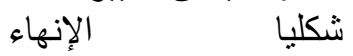

(5)

$$
\text { لوظيفي المنولد، وهذه المر احل هي: }
$$

نوليد

مرحلة الإنهاء تعطي هذه المراحل

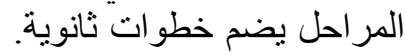
، وكان تصنيف المر احلة

(5)

مر احل تولبد أنماط المساجد الجامعة

\begin{tabular}{|c|c|c|c|c|c|c|c|}
\hline \multirow{2}{*}{$\mathrm{BC}$} & \multirow{2}{*}{\multicolumn{2}{|c|}{ 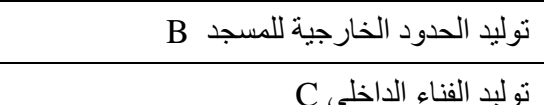 }} & \multirow{2}{*}{ A } & \multirow{2}{*}{\multicolumn{3}{|c|}{ تحديد الحدود العامة لثنكل المسجد وفضاءاته }} & \multirow{2}{*}{1} \\
\hline & & & & & & & \\
\hline \multirow{5}{*}{ PS } & P ت Pديد المصلى P & \multirow{2}{*}{\multicolumn{3}{|c|}{ تحديد المصلى و الفضـاءات الثانوية }} & \multirow{2}{*}{ A } & \multirow{5}{*}{ الثانية الئل المصلى } & \multirow{5}{*}{2} \\
\hline & تحديد الفضاءات الثانوية S & & & & & & \\
\hline & Pقسيم قاعة الصلاة P P & \multirow[t]{2}{*}{ فضـاءاتهما } & \multirow{2}{*}{\multicolumn{2}{|c|}{ تالساسيم المصلى و الفضاءات الثانوية }} & \multirow{2}{*}{ B } & & \\
\hline & S تقسيم الفضاءات الثنانوية S & & & & & & \\
\hline & \multicolumn{2}{|c|}{ فضاءات ثانوية اصغر } & \multicolumn{2}{|c|}{ الأساسية بتقسيمها } & \multirow[t]{2}{*}{$\mathrm{C}$} & & \\
\hline TE & & & & & & الإنهاء & 3 \\
\hline
\end{tabular}

من الضروري توضيح بعض الملاحظات المرتبطة بالناحية الثكلية للأحكام كما يأتي:

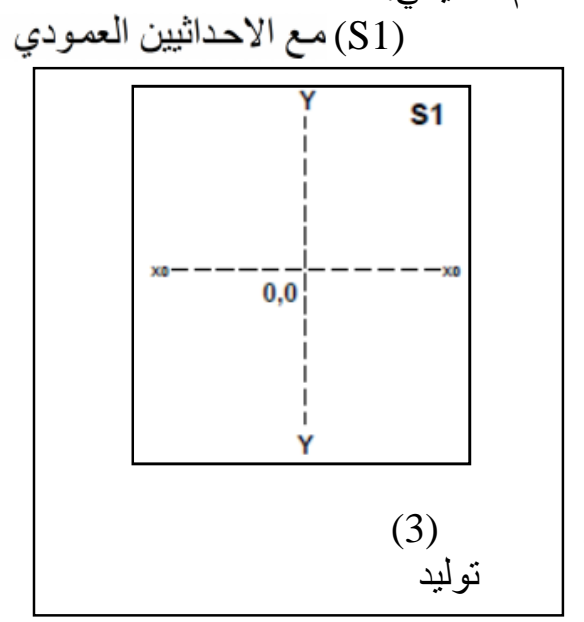

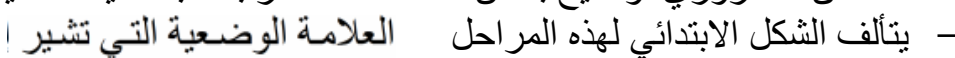
توليد

الجامعة بعناصر ها الحيزية:

التوليدية مابين الأ الإلز امية التي يشترط تنفيذها والأ

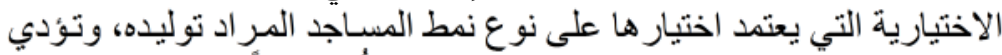

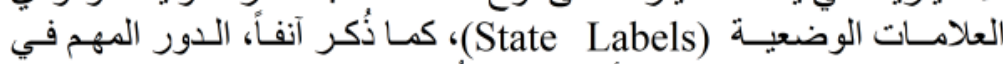

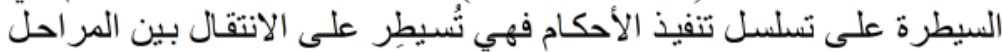

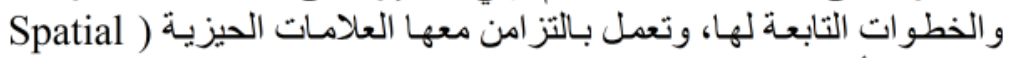
(Labels

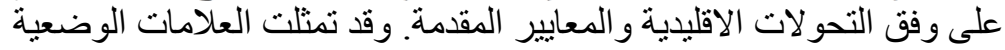

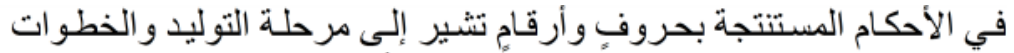

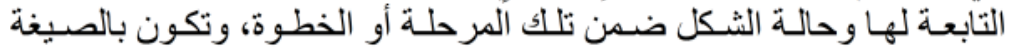

الآتية: (Sn)

(Stage) :S

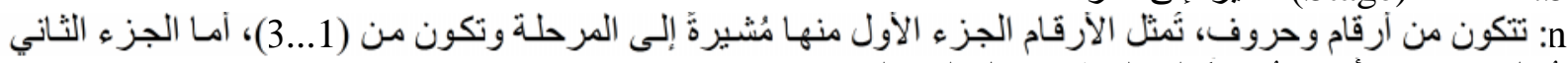

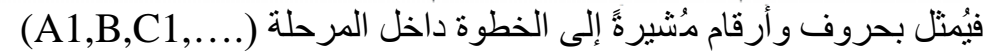

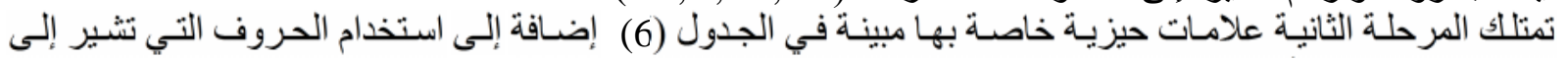


Dec. 2014

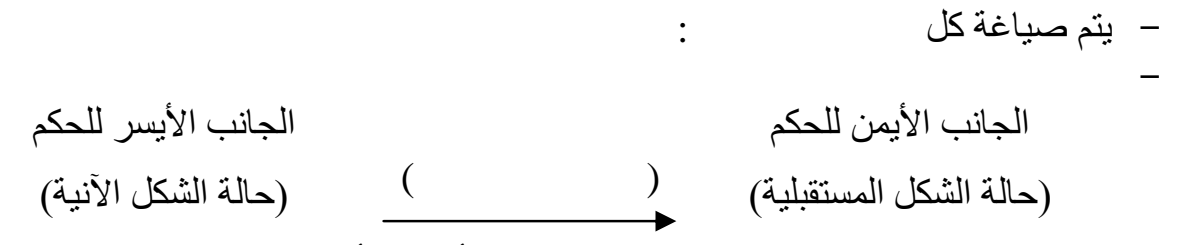

الثكلية للأنماط الأساسية للمساجد الجامعة على وفق مر احلهاو هي:

\begin{tabular}{|c|c|c|c|c|}
\hline وظيفة المؤشرات الثكلية والوظيفية & المؤشرات & 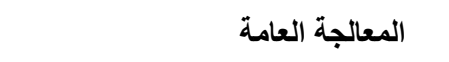 & & المرحلة \\
\hline تقسيم قاعة الصلاة و الفضـاءات الثانوية & & تقسيم المصلى والفضاءات الثانوية إلى & \multirow{3}{*}{ تواليّ المصلى أثاتى } & \multirow{3}{*}{ 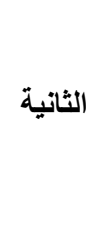 } \\
\hline تقسيم المصلى و الفضـاءات الثانوية & & \multirow{2}{*}{ معالجة الفضاءات الأساسية } & & \\
\hline إضافة عناصر حيزية & & & & \\
\hline
\end{tabular}

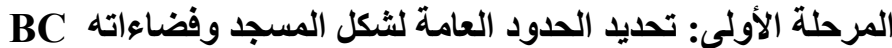

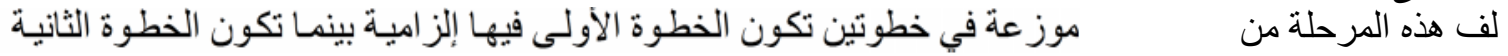
الآتية:

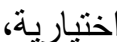

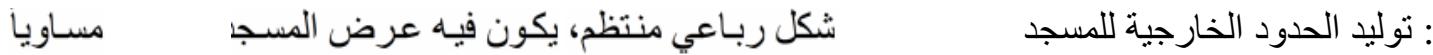

ويتمثل هذا الحكم بالصيغة الآتية (WC1Rec)

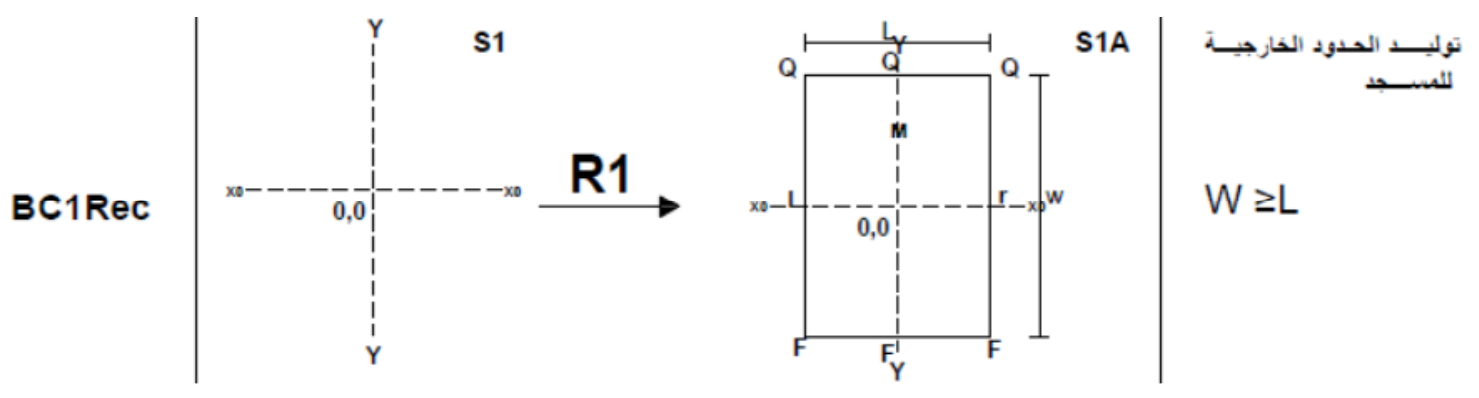

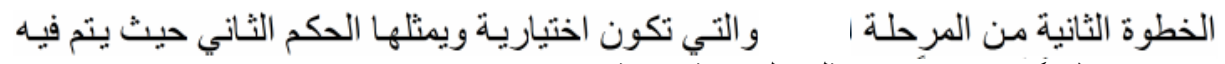
ويكون مسنطيلا ويتمثل بالصيغة الاتية (BC3Rec) :

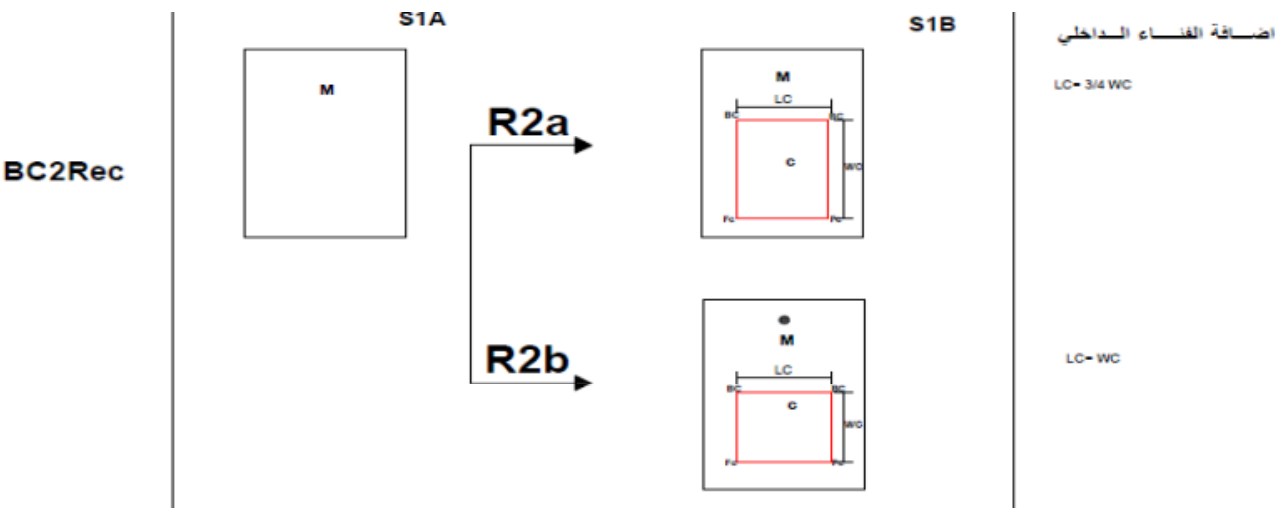




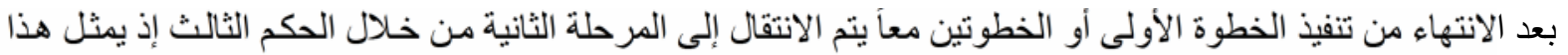

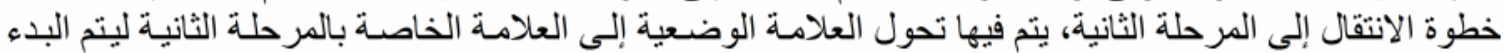

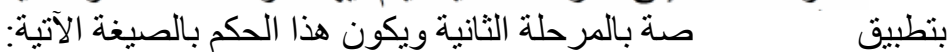
BC3SL:
S1A,S1B
R3
S2

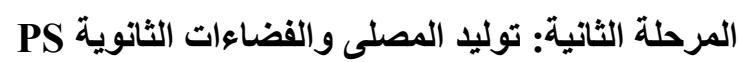

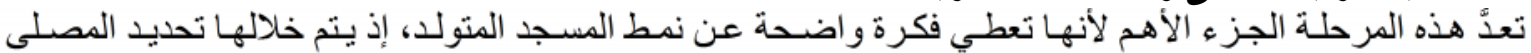

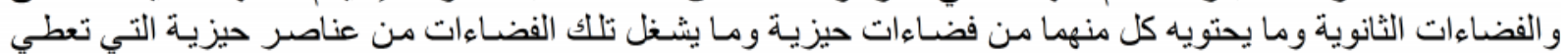

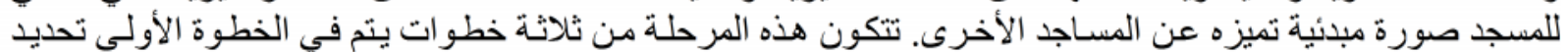

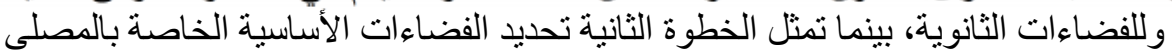

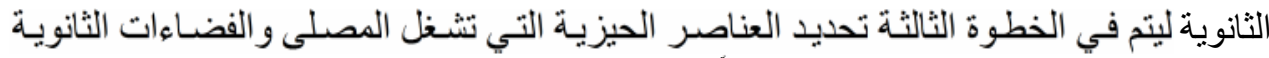

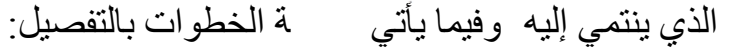

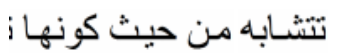

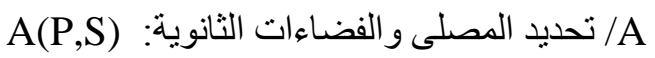

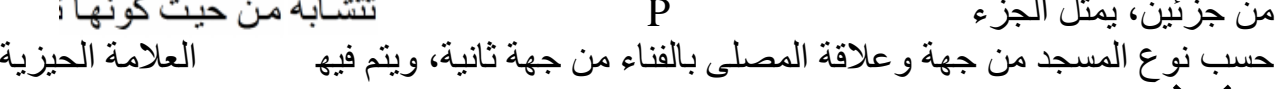

في المساجد غير ذات الفناء

يتن
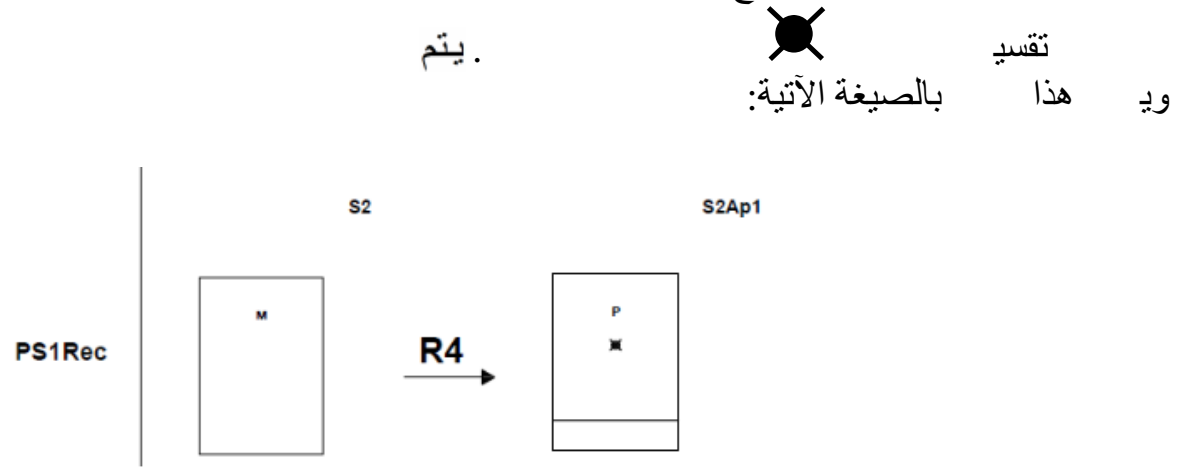

المصلى في المساجد ذات الفناء ولكنه لا يرتبط بشكل مباشر مـع الفناء يفصلهما الرواق

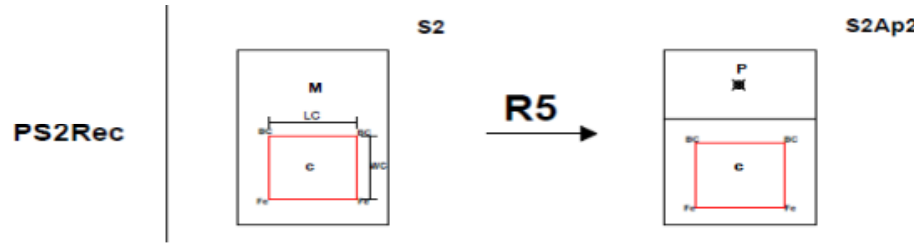

وبينما يتم ب بيغة الآتية:

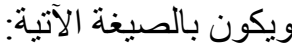

في حين يمثل الحكم الثالث

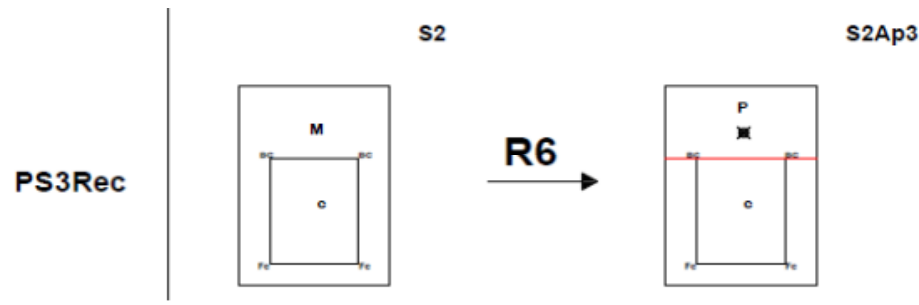

المتمثل بتحديد الفضـاءات الثانويـة الأفقيـة والر أسية،ويتم فيها أيضـا 'لفضاءات الثانويـة

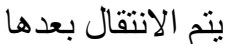
العلامة الحيزية الخاصة بتقسيم 


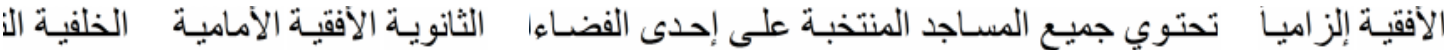

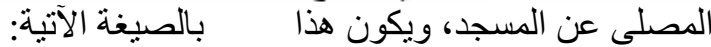

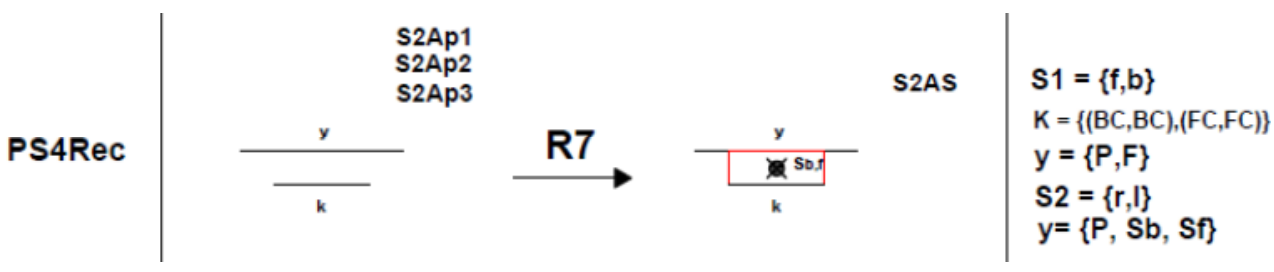

ـ ـ الخطوة الثانية B/ تقسيم المصلى و الفضاءات الثنانوية إلى فضاءاتهما الأساسية:

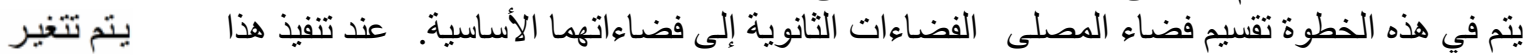

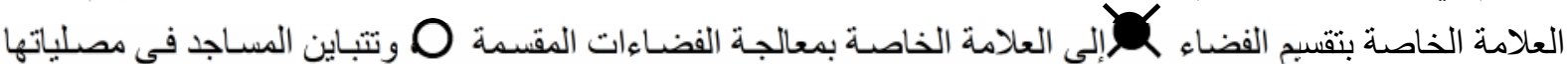

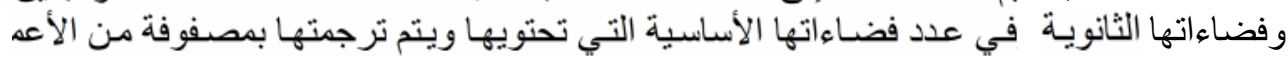
صنيخة هذاء وفينات

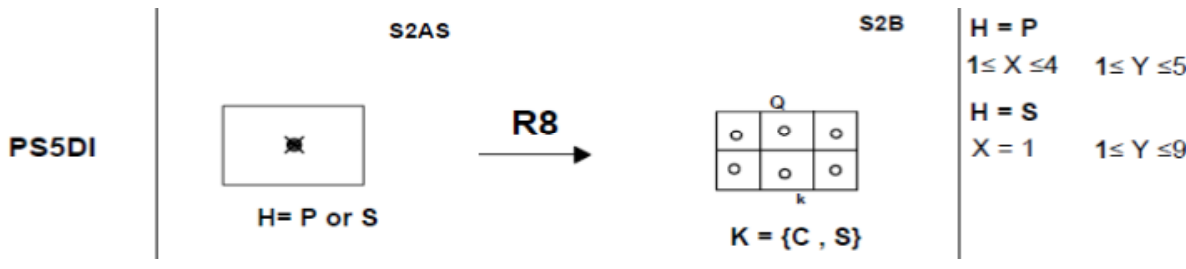

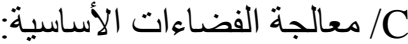

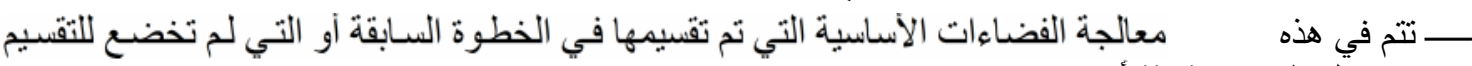

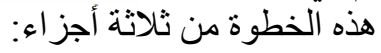
- زءء الأول C1: يُعنى بمعالجة الخلايا الو اقعة على المحاور سو اء كانت مؤلفة من خلية مفردة وهي الخلية الأساسية

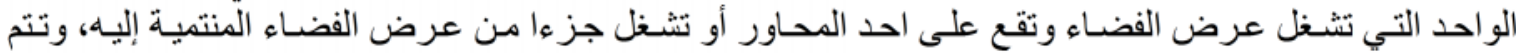

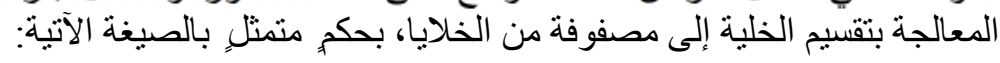

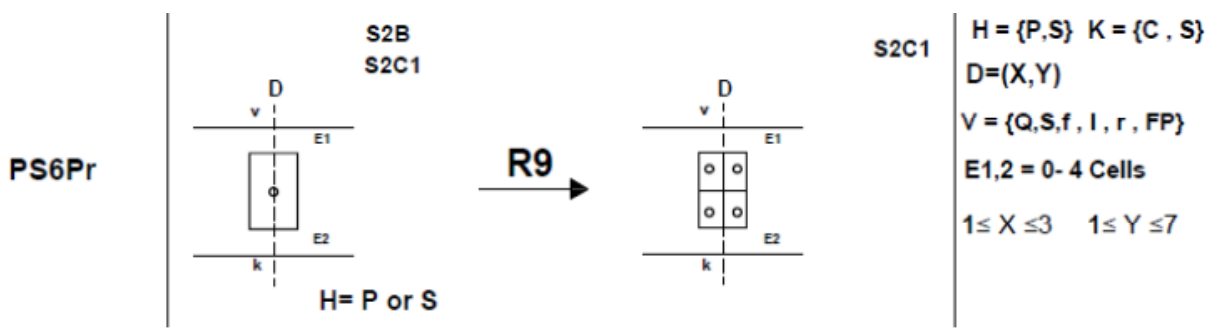

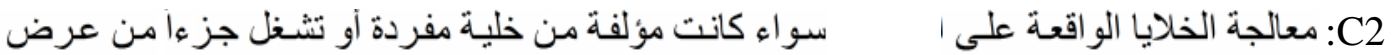

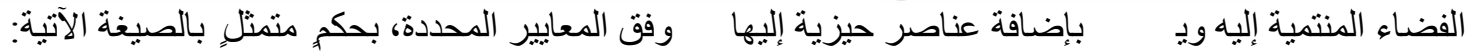




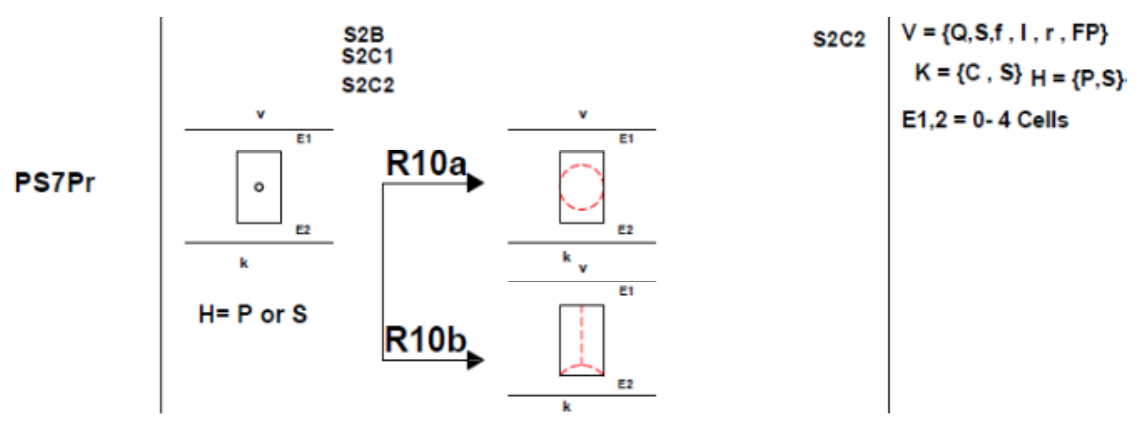

C3: يتم في هذا الجزء معالجة الخلايا المجاورة لخلايا تمت معالجتها مسبقاً وتتم هذه المعالجـة بإضـافة احد

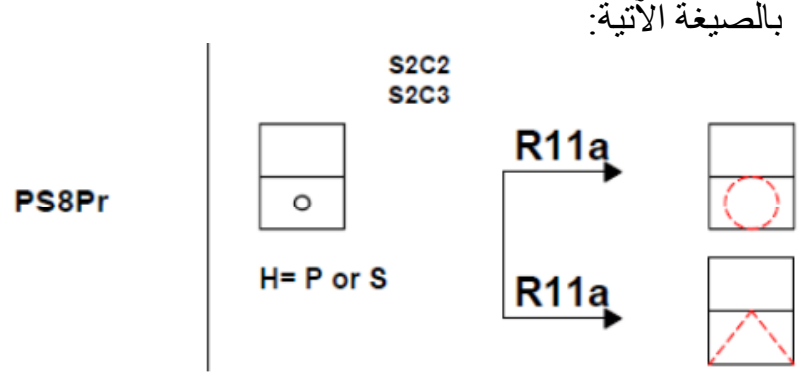

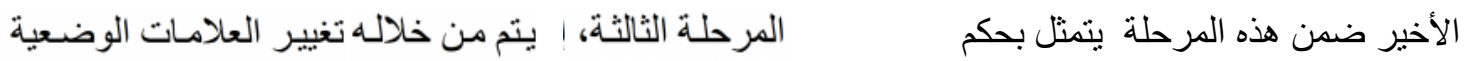
الخاصة بالمرحلة الثانية العلامة الوضعية التي تؤشر بداية المرحلة الثالثة وهي مرحلة الإنهاء. ويتم

PS9SL: $\quad$ S2AS, S2C2,S2C3 $\stackrel{\mathrm{R} 12}{\longrightarrow} \mathrm{S} 3$

تمثيل هذا الحكم

TE "Termination" المرحلة الثالثة: مرحلة الإنهاء

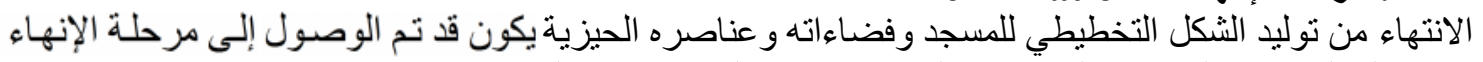

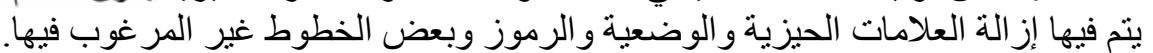

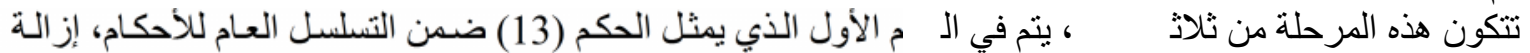

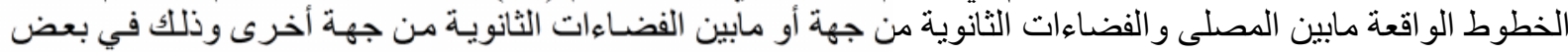

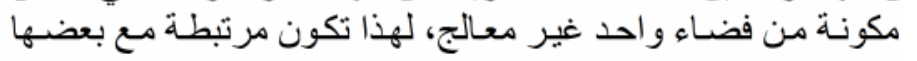

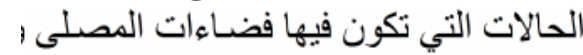

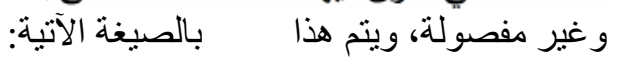

\section{TE1De : $\quad$ S3 $\stackrel{\text { R13 }}{\longrightarrow}$ S3A}

$$
\text { بفضاءات المسجد ويكون بالصيغة الآتية: }
$$

بينما يتم في الـ

TE2De : $\mathrm{S} 3, \mathrm{~S} 5 \mathrm{~A} \stackrel{\mathrm{R} 14}{\longrightarrow} \mathrm{S} 3 \mathrm{~B}$

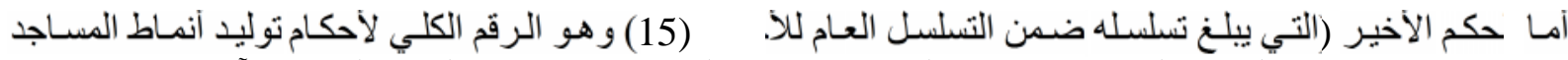

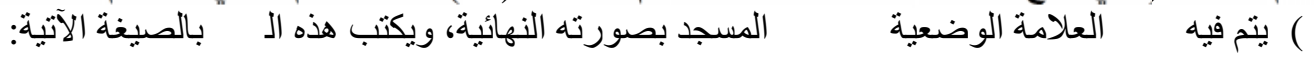

TE3De : $\quad$ S3B $\stackrel{\mathrm{R} 15}{\longrightarrow} \#$

طوات داخل المر احل وتحرك المك وكي

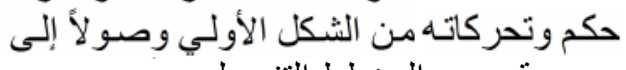

عة حسب المخطط التفصبا

\author{
وتسلسلها وكيفية تحركها ضمن \\ خلالها إذ يثير الجانب الأيمن منها \\ حكم النهائي ينما يشير الجير لجانب الأيسر منها توليد
}




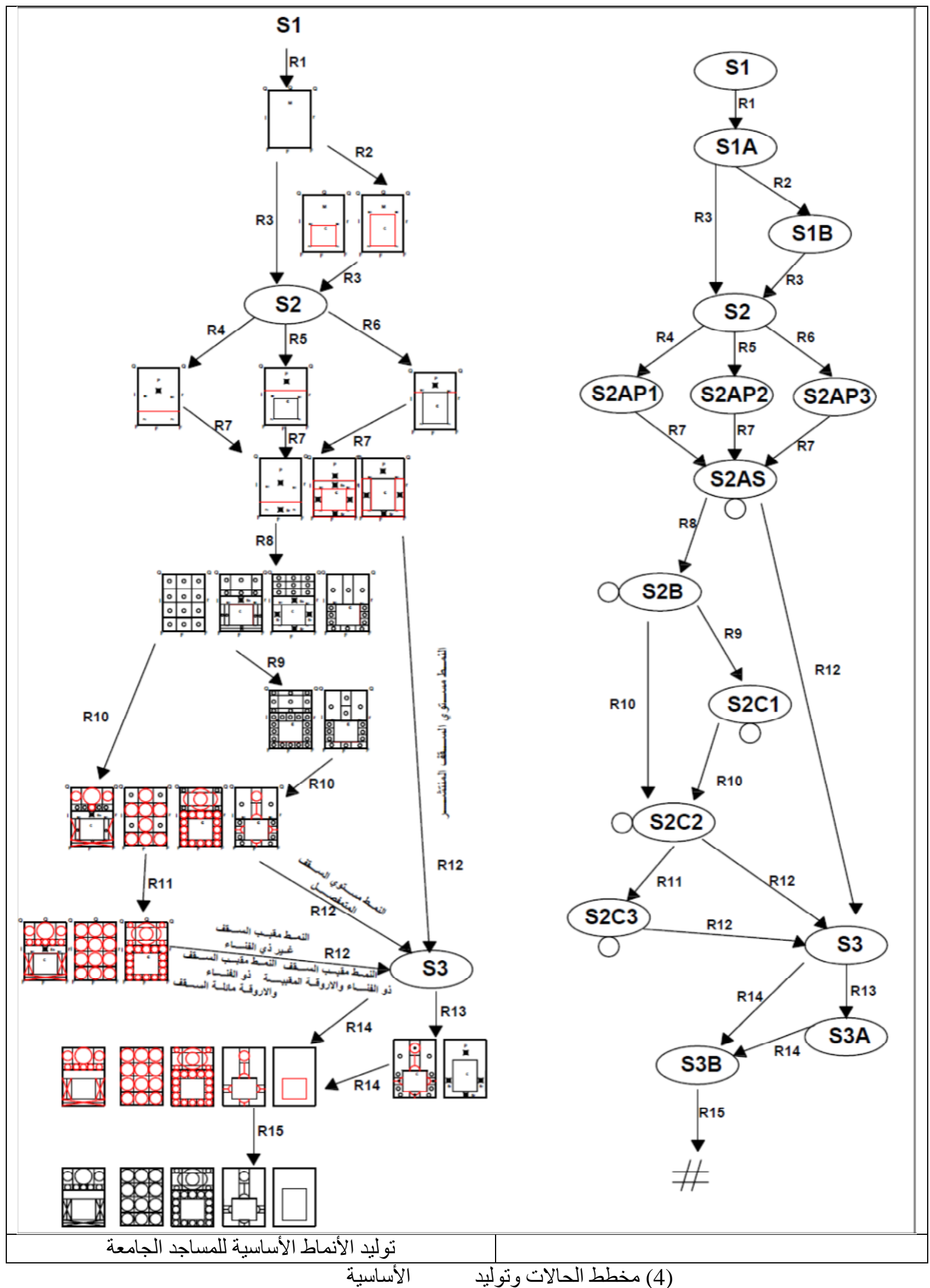




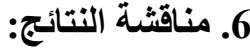

تتضمن هذه الفقرة مناقتة الإجر اءات العملية التي تمت بها تحديد وصياغة الأ المستنتجة للأنماط الرئيسة للمساجد

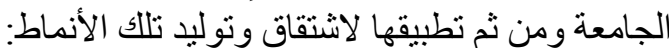

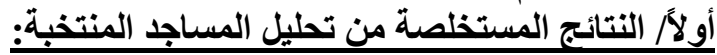

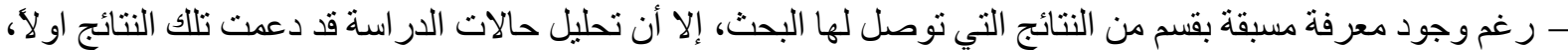

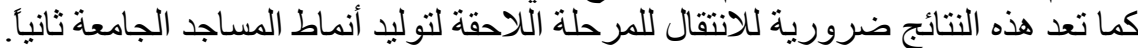

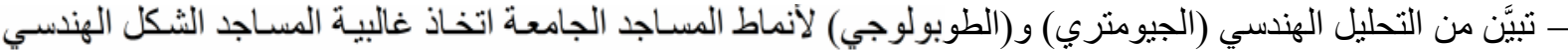

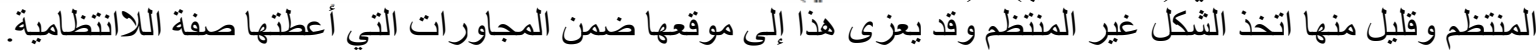

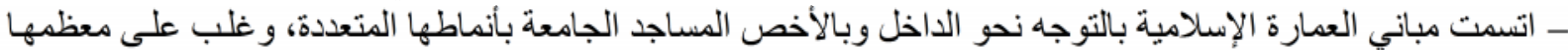

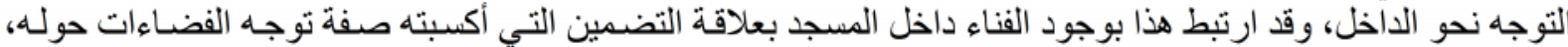

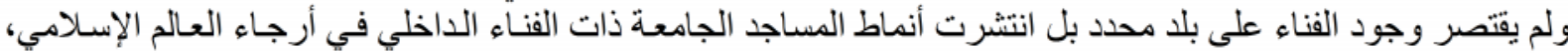

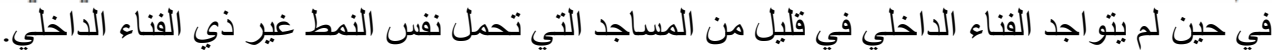

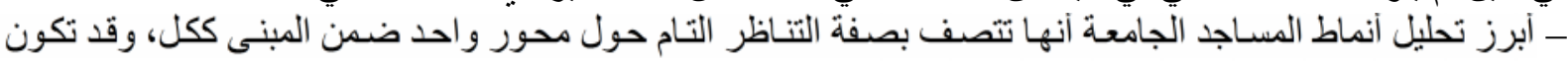

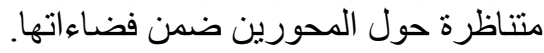

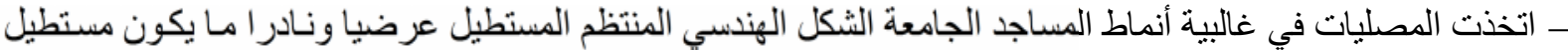
طولياً، وتكون علاقته متجاورة بصورة مباشرة أو غير مباشرة مع الفناء.

الرئيسة للمسـاجد الجامعة بلغ (15) ويعزى هذا

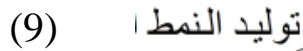

أ، في حين تـ النمط مقبب السقف

(12) حكمـا مـن مجمـوع (15) حكمـا. وفيمـا يلـي
ثثاتياً/ النتائج الخاصة بقواعد توليد أنماط المساجد الجامعة:

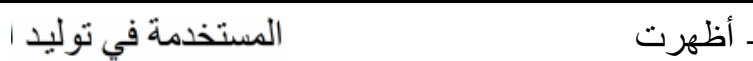

تباينها اشتر الك غالبية دمة لتوليدها،

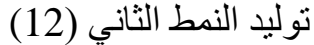

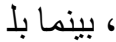

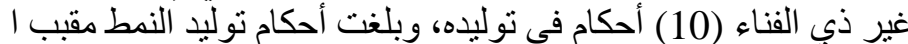

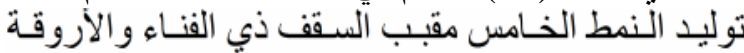

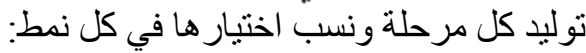

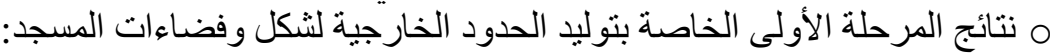

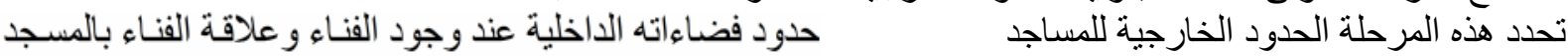

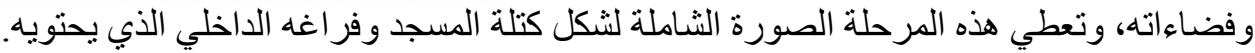

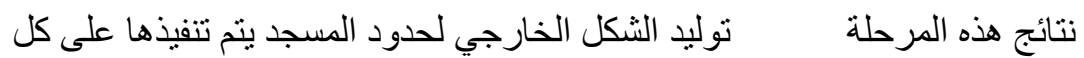

المساجد الجامعة و الذي بتخذ

- بيَّن توليد الفناء الداخلي، انه بمكن تتفيذه

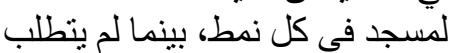

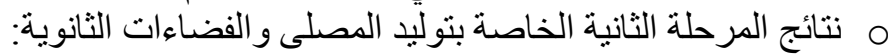

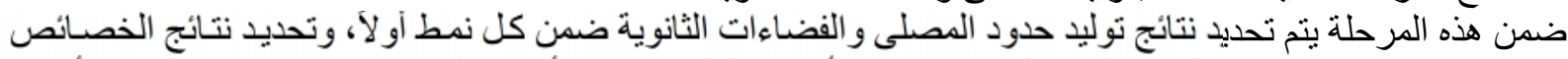

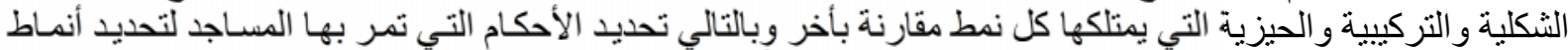

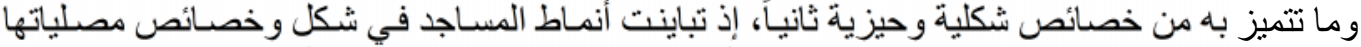

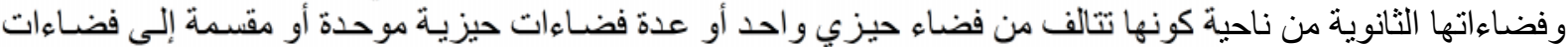

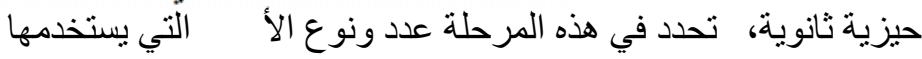

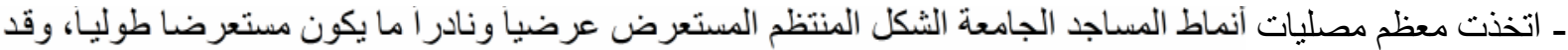

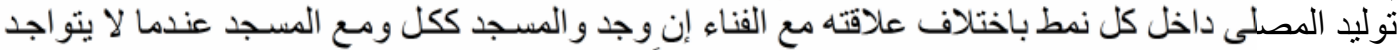

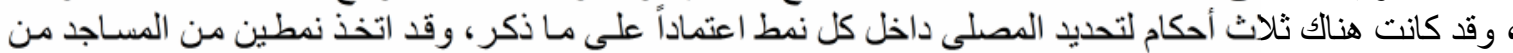

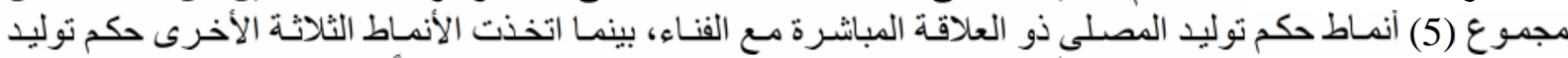

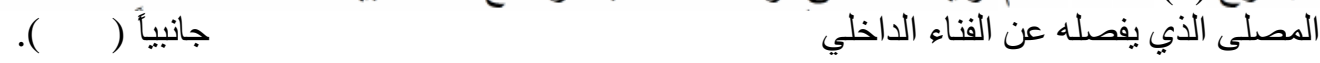

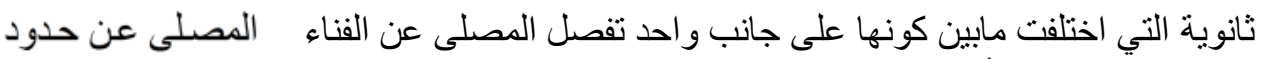

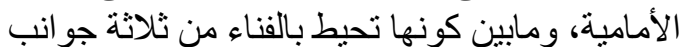

أي كان موقعهة، بينمـا اتخذ نمطين أيضـا توليد الفضاء

الأمامية

اتخذ نمطي 
توليد الثكل التخطيطي للفضـاءات الثانويـة (U Shape) و الذي يحبط بالفناء من ثُلاث جهات، و اتخذ نمطين آخرين

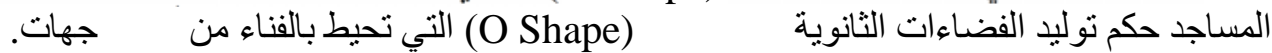

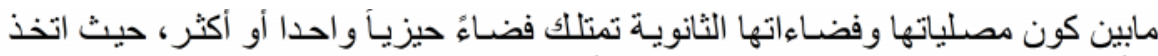

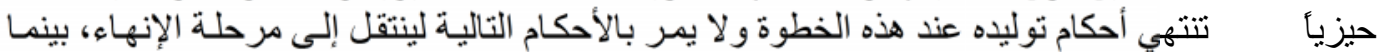

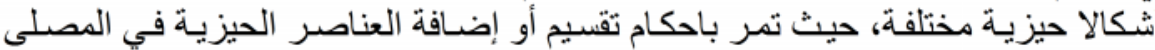

$$
\text { و الفضاءات الثانوية }
$$

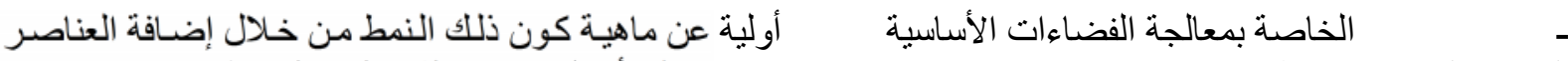

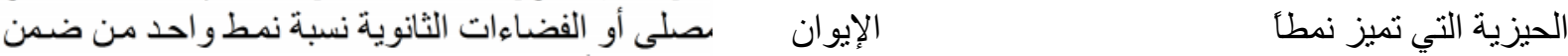

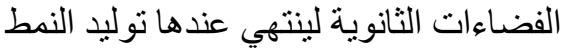

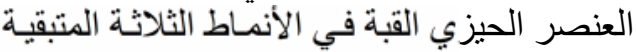

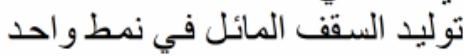

$$
\text { المصلى فقط ضمن تلكئك }
$$

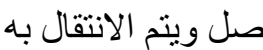

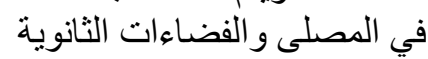

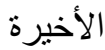

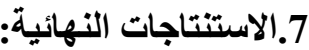

الاستنتاجات النهائية للبحث من خلال تطبيق الأ. تبينت الإستنتاجات الآتية:

بعد الانتهاء من التطبيق واستعر

هُ هب عبارة عن نتائج

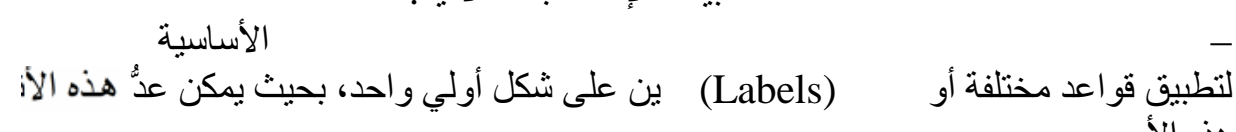

\section{الأساسية}

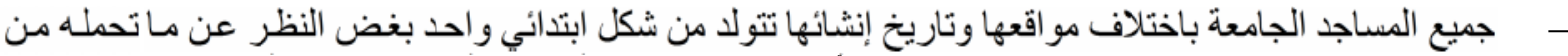

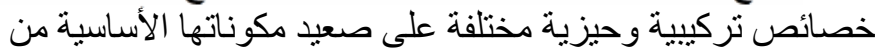

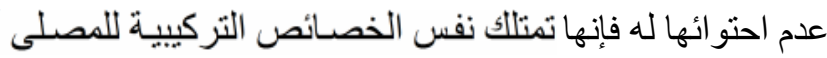

$$
\text { الثانوية لغة تصميمية واحدة أمكن في هذا البحث تحديد طبيعتها. }
$$

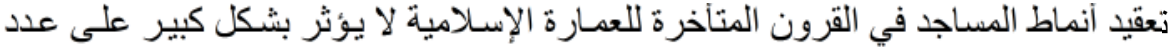

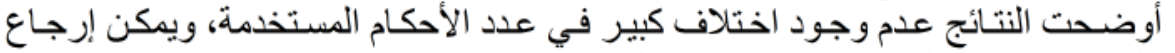

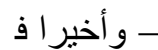

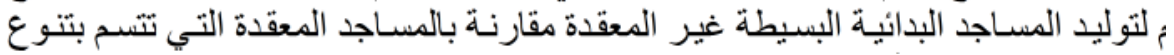

حكام المستخدمة لتوليده

$$
\text { الإسلامية. }
$$

وتعدد الفضاءات الحيزية التي ظهرت في القرون

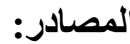

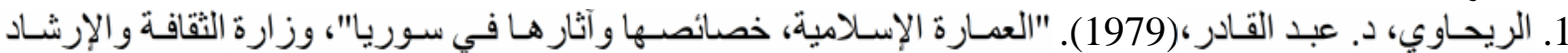

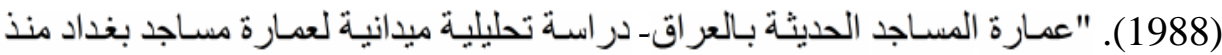

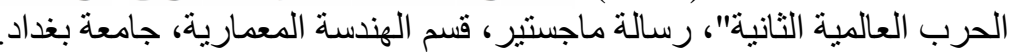

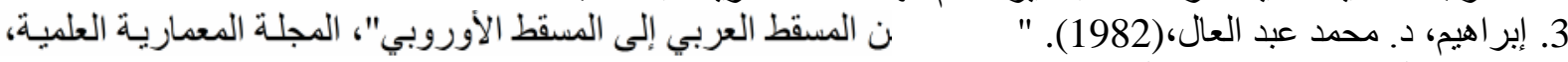

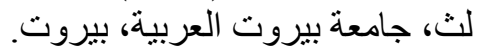
.37

4. شافعي، د. فريد، (1970). "العمارة العربية في مصر الإسلامية"، الهيئة المصرية للتأليف و النشر، القاهرة.

" حسين، (1981).

6. Ahmad, S. \& Chase, (2004). "Design Generation of Central Asian Caravanserai. Use of a Parametric Shape Grammar for the Analysis of Historic Islamic Architecture", 1st ASCAAD International Conference, e-Design in Architecture, KFUPM, Dhahran, Saudi Arabia. December.

7. AL- Kazzaz, D. A. A., (2011). "Shape Grammars for Hybrid Component- Based Design", Ph.D Dissertation, University of Strathclyde, Glasgow, Scotland, United Kingdom.

8. Ardalan, Nader, (1980). "A Preliminary Study of Mosque Architecture" in Architecture as Symbol and Self Identity, the Aga Khan Award for Architecture, Philadelphia.

9. Eilouti. Buthayna H. \& Amer M. Al-Jokhadar, (2007). “A Generative System for Mamluk Madrasa Form-Making". Nexus Network Journal-VOL.9, NO.1, 7-30. 
10. Eilouti. Buthayna H. \& Amer M. Al-Jokhadar, (2007). "A Computer-Aided Rule-Based, Mamluk Madrasa Plan Generator". Nexus Network Journal-VOL.9, NO.1, 31-58.

11. Eilouti. Buthayna H. \& Al Shaar, Mohammed Jamil Hamamieh, (2012). "Shape Grammar of Traditional Damascene Houses". International, Journal of Architectural Heritage: Conservation, Analysis and Restoration.

12. Cagdas, Gulen, (1996)."A Shape Grammar: The Language of Traditional Turkish Houses".Istanbul Technical University, Faculty of architecture, Istanbul, Turkey.

13. Chase S. C., (1996). "Modeling Designs with Shape Algebras and Formal Logic", Ph.D Dissertation, University of California, Los Angeles.

14. Chen, X., (2005)."Relationships Between Product Form and Brand: A Shape Grammatical Approach". University of Leeds, Leeds.

15. Colakoglu, M. Birgul, (2000). "Design by Grammar: Algorithmic Design in an Architectural Context", Ph.D Dissertation, Yildiz Technical University.

16. Creswell, K.A.C. "Short Account of Early Muslim Architecture" Offset Conrogravure, Berirut, Lebanon, (1968).

17. Duarte, J. P. (2001)."Customizing Mass Housing: a Discursive Grammar for Siza's Malagueira Houses". Ph.D Dissertation, Massachusetts Institute of Technology, Cambridge, MA.

18. Duarte,J.P.\& Eloy,S. (2012)." A Transformation Grammar-Based Methodology for Housing Rehabilitation", Design Computing and Cognition DCC'12. J.S.

19. Flemming, U. (1987)."More Than the Sum of Parts: the Grammar of Queen Anne Houses". Environment and Planning B: Planning and Design, 14, 323-350.

20. Gero, J.S. \&Jo, J.H., (1995). "Space Layout Planning Using an Evolutionary Approach", Artificial Intelligence in Engineering, 12(3).

21. Hillenbrand, R., (1994). "Islamic Architecture, Form, Function and Meaning", Edinburgh University Press.

22. Hoag, J., (1979). "Islamic Architecture”, Harry N. Abrams, Inc. Publisher, New York.

23. Knight, T.W., (1981). "Languages of Design", Environment and Planning B. PION Publication, London, Vol.8, P213-238.

24. Knight, T. W. (1994)."Transformations in Design: A Formal Approach to Stylistic Change and Innovation in the Visual Arts". Cambridge: Cambridge University Press.

25. Loomis, Benjamin, (2002). "A User-Driven Genetic Algorithm for Eevolving NonDeterministic Shape Grammars", Massachusetts Institute of Technology, Cambridge MA, USA.

26. Michalek, J., (2001). "Interactive Layout Design Optimization", MS thesis, University of Michigan,

27. Michell, G. \& Grube E., (1978). “Architecture of the Islamic World”, Thames \& Hudson Ltd., London.

28. Orsborn, S., Cagan, J., Pawlicki, R., \& Smith, R. C. (2006)."Creating Cross-Over Vehicles: Defining and Combining Vehicle Classes Using Shape Grammars". Artificial Intelligence for Engineering Design, Analysis and Manufacturing, 20, 217-246.

29. Prochazka A., (1986). "Mosques", in Architecture of the Islamic Cultural Sphere, MARP, Muslim Architecture Research Program, Zurich.

30. Sener \& Gorgul (2008)" A Shape Grammar Algorithm and Educational Software to Analyze Classic Ottoman Mosques". ITU A|Z VOL: 5 NO: 1.

31. Steadman, Philip, (1983). "Architecture Morphology", Pion Ltd., London.

32. Stiny, G., (1977), "Ice-Ray: A Note on the Generation of Chinese Lattice Designs". Environment and Planning B: Planning and Design, 4, 89-98. 
33. Stiny,G., (1978).“An Evaluation of Palladian Plans”, Environment and Planning B, vol.5, PP.199-206.

34. Stiny G., (1980a). "Introduction to Shape and Shape Grammars" Environment and Planning B: Planning and Design 7 343-351.

35. Stiny G., (1980b). "Kindergarten Grammars: Designing with Froebel's Building Gifts" Environment and Planning B: Planning and Design 7 409-462,

36. Stiny, G. (1985)."Computing with Form and Meaning in Architecture". Journal of Architectural Education, 39(1 autumn), 7-19.

37. Stiny, G. \& Mitchell, J., (1978). "The Palladian Grammar", Environment and Planning B, vol.5, pp.5-11.

38. Ulu and Sener, (2009)."A Shape Grammar Model to Generate Islamic Geometric Pattern". Generative Art Conference GA 2009, Istanbul, Turkey.

تم اجراء البحث في كلية ألهندسة = جامعة ألموصل 